\title{
Exploration of Prognostic Immune-Related Genes and lncRNAs Biomarkers in Kidney Renal Clear Cell Carcinoma and Its Crosstalk with Acute Kidney Injury
}

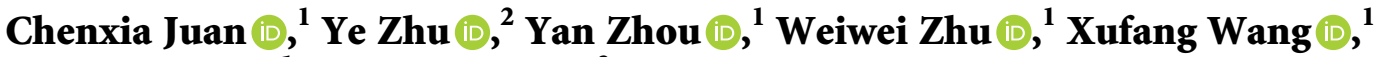 \\ Weiming $\mathrm{He} \mathbb{D}^{1},{ }^{1}$ and Yan Chen $\mathbb{D}^{3}$ \\ ${ }^{1}$ Department of Nephrology, Jiangsu Province Hospital of Chinese Medicine, \\ Affiliated Hospital of Nanjing University of Chinese Medicine, Nanjing, Jiangsu, China \\ ${ }^{2}$ Department of Nephrology, Second Affiliated Hospital of Soochow University, Suzhou, Jiangsu, China \\ ${ }^{3}$ Department of Nephrology, Jiangsu Province Geriatric Hospital, Jiangsu Province Official Hospital, Nanjing, Jiangsu, China
}

Correspondence should be addressed to Weiming He; mousehwm@163.com and Yan Chen; cheny@njmu.edu.cn

Received 2 December 2021; Accepted 5 January 2022; Published 8 February 2022

Academic Editor: Song Cao

Copyright $\odot 2022$ Chenxia Juan et al. This is an open access article distributed under the Creative Commons Attribution License, which permits unrestricted use, distribution, and reproduction in any medium, provided the original work is properly cited.

Kidney renal clear cell carcinoma (KIRC) has a poor prognosis and a high death rate globally. Cancer prognosis is strongly linked to immune-related genes (IRGs), according to numerous research. We utilized KIRC RNA-seq data from the TCGA database to build a prognostic model incorporating seven immune-related (IR) lncRNAs, and we constructed the model using LASSO regression. Additionally, we calculated a risk score for each patient using a prognostic model that divided patients into high-risk and low-risk groups. The ESTIMATE and CIBERSORT methodologies were then used to analyze the differences in the tumor microenvironment of the two groups of patients. Finally, we predicted three small molecule drugs that may have potential therapeutic effects for high-risk patients. We combined the acute kidney injury dataset to obtain differential genes that may serve standard biological functions with two risk groups. Our study shows that the model we constructed for IR-lncRNAs has reliable predictive efficacy for patients with KIRC.

\section{Introduction}

Kidney renal clear cell carcinoma (KIRC) is a common tumor, accounting for $70-80 \%$ of all kidney tumors [1]. Every year, around 210,000 new cases of KIRC are diagnosed throughout the globe [2]. Despite significant progress in recent years in understanding the mechanisms and treatments of KIRC, the prognosis for KIRC patients remains poor [3]. For instance, the 5-year overall survival (OS) rate for stage I KIRC patients is around $80 \%-95 \%$, whereas the 5 year OS rate for stage IV KIRC patients is less than 10\% [4]. As a result, new biomarkers and therapy alternatives are urgently needed to assist doctors in identifying suitable treatment options and medicines and more correctly predicting the prognosis of KIRC patients.
Tumor immunity has attracted scientists' attention as a result of advances in the research of various cancers. Immune-related genes (IRGs) are thought to have a role in tumor growth and progression $[5,6]$. In various cancers, immunotherapy that targets PD-1 ligand (PD-L1)/programmed death 1 (PD-1) signalling, for example, has been demonstrated to increase antitumor immunity [7]. Furthermore, studies have shown that PD-L1 and PD-1 expressions in KIRC patients are higher and correlate with patient prognosis and that immune checkpoint inhibitor drugs provide better options for KIRC patients, such as improved OS and chemotherapy tolerance [8]. Tumor microenvironment (TME) research is also becoming more critical in the development of tumors [9]. The TME comprises extracellular matrix components, immune cells, 
stromal cells, and inflammatory mediators [10]. One of the primary cell types in the TME, immune infiltrating cells, is strongly associated with tumor therapeutic response [11]. Using immune-based prognostic markers in KIRC is a promising method, according to the results of these studies $[12,13]$. Furthermore, the findings of this research might be utilized to investigate the underlying biological processes further and identify possible therapeutic medications that could help KIRC patients have a better prognosis.

Through transcription regulation, long noncoding RNAs (lncRNAs) have been shown to have a role in the creation and activation of a variety of immune cells, as well as tumor metastasis [14, 15]. Immune-related lncRNAs (IR-lncRNAs) can be used as markers to predict the prognosis of glioma patients [16]. It has been shown that creating IR-related models may predict the prognosis of patients with various kinds of cancers, including lung cancer, glioblastoma, and gastric cancer [17-19]. However, the prognostic significance of IRGs and IR-lncRNAs for KIRC is currently being researched. We analyzed the relevance of IRGs and IRlncRNAs in predicting the prognosis of KIRC patients in this work. We used least absolute shrinkage and selection operator (LASSO) regression to develop a model incorporating seven IR-lncRNAs for predicting overall survival. Based on the model, risk scores were produced for each patient, and patients were split into low- and high-risk groups. The TME of the samples was then examined by utilizing ESTIMATE and CIBERSORT algorithms to calculate the quantity of stromal and immune cells in every sample. Finally, we identified three small-molecule medicines that might be used to treat individuals at high risk of KIRC. We also looked at the AKI dataset to see whether any genes had both interactions with KIRC. We found that the model we constructed consisting of IRGs and IR-lncRNA was able to predict the prognosis of KIRC patients.

\section{Materials and Methods}

2.1. Data Collection. We downloaded data from the TCGA (The Cancer Genome Atlas) database for 537 RNA-seq cases, containing clinical information of the cases. We downloaded the RNA-seq dataset containing 39 human kidney biopsy samples (AKI group) and 9 reference nephrectomy groups (REF) from GEO (Gene Expression Omnibus) (GSE139061). Clinical features of 537 samples are shown in Table 1. The latter study included patients with KIRC who had complete clinical data and RNA-seq data. ImmPort Shared Data provided us with a total of 2483 IRGs (Table S1).

\subsection{WGCNA Network Construction. To discover modular} genes associated with clinical features, we used the weighted gene coexpression network analysis (WGCNA) approach to construct a network [20]. The gene-gene association is determined for each pair in the first stage using gene coexpression similarity. The adjacency matrix and topological overlap matrix (TOM) are then constructed using "soft" criteria through the adjacency function. Using a hierarchical clustering of heterogeneity measure (1-TOM) method, each
TABLE 1: Clinicopathological parameters of KIRC patients in this study.

\begin{tabular}{|c|c|c|c|}
\hline Variables & Subgroups & $N$ & $\%$ \\
\hline \multirow{2}{*}{ Age } & $\leq 65$ & 352 & 65.55 \\
\hline & $>65$ & 185 & 34.45 \\
\hline \multirow{2}{*}{ Gender } & Male & 346 & 64.43 \\
\hline & Female & 191 & 35.57 \\
\hline \multirow{5}{*}{ Grade } & G1 & 14 & 2.61 \\
\hline & G2 & 230 & 42.83 \\
\hline & G3 & 207 & 38.55 \\
\hline & G4 & 78 & 14.53 \\
\hline & Unknown & 8 & 1.48 \\
\hline \multirow{4}{*}{$\mathrm{T}$ classification } & $\mathrm{T} 1$ & 275 & 51.21 \\
\hline & $\mathrm{T} 2$ & 69 & 12.85 \\
\hline & $\mathrm{T} 3$ & 182 & 33.89 \\
\hline & $\mathrm{T} 4$ & 11 & 2.05 \\
\hline \multirow{3}{*}{$\mathrm{N}$ classification } & No & 240 & 44.69 \\
\hline & N1 & 17 & 3.17 \\
\hline & Unknown & 280 & 52.14 \\
\hline \multirow{3}{*}{ M classification } & M0 & 426 & 79.33 \\
\hline & M1 & 79 & 14.71 \\
\hline & Unknown & 32 & 5.96 \\
\hline \multirow{5}{*}{ UICC stage } & Stage I & 269 & 50.09 \\
\hline & Stage II & 57 & 10.61 \\
\hline & Stage III & 125 & 23.28 \\
\hline & Stage IV & 83 & 15.46 \\
\hline & Unknown & 3 & 0.56 \\
\hline \multirow{2}{*}{ Survival status } & Alive & 360 & 67.04 \\
\hline & Dead & 177 & 32.96 \\
\hline
\end{tabular}

"gene module" is a set of genes with a high degree of topological overlap.

Pearson's technique was used to find correlations between modules and clinical variables to find clinically relevant modules. We then chose modules that had a strong association with prognostic characteristics.

2.3. Identification of Prognosis-Related IRG and IR-IncRNAs. All of the IRGs in the prognosis-related module were subjected to univariate Cox regression analysis, and only those with a $P$ value $<0.05$ were recognized as prognosisrelated. We then used Pearson correlation analysis to determine the correlations between prognostic IRGs and all lncRNAs in KIRC patients; correlations more than 0.6 were designated as IR-lncRNAs. Finally, all IR-lncRNAs were submitted to univariate Cox regression, and lncRNAs with $P<0.001$ were selected as prognostically relevant IRlncRNAs.

2.4. Establishment of Prognostic Signature. We used LASSO Cox regression to analyze all prognosis-related IRG and IRlncRNAs to construct a prognostic model. The following equation was used to obtain the model risk scores:

$$
\text { Risk score }=\sum_{i=1}^{n} \beta i * x i,
$$


where $x i$ is the gene expression value and $\beta i$ reflects the coefficient produced from LASSO Cox regression analysis; based on the median values of the calculated risk scores, we divided the KIRC patients into two groups of high risk and low risk. To validate the model, we had three cohorts, the training set, the validation set (random $1: 1$ assignment), and the total cohort, which were analyzed using Kaplan-Meier's method. To validate the predictive efficacy of the model, we used time-dependent ROC curves, combined with clinical information for univariate and multifactorial analyses.

2.5. Immune Infiltration Analysis. To investigate the relationship between our predictive model and immune cell infiltration, we used the CIBERSORT method [21] to calculate the amount of 22 immune cells in each KIRC sample; we also used the Wilcoxon rank-sum test to compare the differences in immune cells between high- and low-risk groups and then used the K-M method to look at the relationship between immune cell infiltration and the OS of KIRC patients.

2.6. Pathway Enrichment Analysis and Identification of DEGs. We used the edgeR package to analyze the differentially expressed genes (DEGs) in the two groups. We used CMap, a database of small molecule drugs (https://portals. broadinstitute.org/cmap), set $|\log 2 \mathrm{FC}| \geq 1$, and finally calculated an enrichment score. A positive score suggests that the drug may raise the risk of mortality in people with KIRC. A negative value implies that the drug may reduce the risk of mortality in individuals with KIRC. A drug with a negative score has the potential to be therapeutic. Using the PubChem database, we constructed two-dimensional structural maps of the prospective small molecule medicines (https:// pubchem.ncbi.nlm.nih.gov/).

2.7. GSEA. We used gene set enrichment analysis to see whether there was a substantial difference in gene set enrichment between the high- and low-risk categories in the MSigDB collection (GSEA) [22]. For each study, 1,000 permutations of gene sets were done.

2.8. Statistical Analysis. We used $R$ software (version 3.8.0) to perform statistical analysis. Risk scores and clinical features were examined using the $\chi^{2}$ test to see whether there was any correlation. The statistical significance of normally distributed variables was compared between the two groups using unpaired $t$-tests. All statistical tests were two-tailed, and statistical significance was defined as a value of $P<0.05$.

\section{Results}

3.1. Construction of a Coexpression Network. A total of 2483 IRGs from 539 KIRC samples were subjected to a WGCNA study. When the threshold value is set to 4 , the gene network's connections form a scale-free network distribution (Figure 1(a)). Then we got six coexpressed modules in various colours (Figure 1(b)). Age, sex, grade, stage, overall survival, and survival status were used to determine the connection between modules and clinical features. The survival status of red and grey modules was discovered to be connected (Figure 1(c)). Consequently, the red and grey modules were selected as potential candidates for further investigation in the upcoming research.

\subsection{Identification of Prognostic-Related IRGs and IR-IncRNAs.} The follow-up survival study includes 526 samples with complete survival data. For all IRGs in the red $(n=74)$ and grey $(n=80)$ modules, a univariate Cox regression analysis was conducted (Tables S2 and S3), and 63 genes with $P<0.05$ were identified as prognosis-associated IRGs (Table S4). Subsequently, correlations $\geq 0.6$ were obtained for 313 IR-lncRNAs with prognostic IRGs. Also, 206 IRlncRNAs had good predictive value with $P<0.001$ in the one-way Cox regression analysis (Table S5).

3.3. Construction of a Predictive Model. We used LASSO regression analysis to screen for efficient prognostic-related markers, and a prognostic model was constructed from the training set (Figure 2). The most helpful predictive biomarkers were discovered as DLGAP1-AS2, AC024060.2, SLBP-DT, DGUOK-AS1, MYG1-AS1, AC00578.1, and MELTF-AS1 (Table 2).

We calculated a risk score for each patient and then divided the patients into low-risk and high-risk groups by a median value of cut-off. The expression levels of biomarkers in each group were also analyzed (Figure 3 ).

3.4. Correlation between Signature and Clinicopathologic Characteristics. Age, gender, class, and N categorization did not vary substantially between the high-risk and low-risk groups in any of the cohorts, as shown in Table 3. The training group and the overall cohort, on the other hand, had substantially different stages, Tclassification, and $\mathrm{M}$ classification.

3.5. Prognostic Value of Signature for Assessing Overall Survival. Patients in the high-risk group have a poorer prognosis, as indicated in Figures 4(a)-4(c). Patients' survival times fell as their risk score rose, as seen in Figures 4(d)-4(f), and more patients died in the high-risk group. The Kaplan-Meier test was used to confirm the model's prognostic usefulness further. Univariate Cox regression analysis supported the model's prognostic usefulness (Figures 5(a)-5(c)). In a multivariate analysis of the whole cohort, the risk score was an independent risk factor for KIRC patient OS (Figures 5(d)-5(f)). The AUC for OS at 1,3 , and 5 years was $0.744,0.695$, and 0.759 in training cohort; $0.651,0.653$, and 0.683 in validation cohort (Figure 5(h)); and 0.700, 0.675, and 0.723 in the total cohort (Figure 5(i)).

3.6. High-Risk Scores Were Related to Higher Immune Scores in KIRC Patients. As seen in Figure 6(a), individuals classified as high risk had significantly higher immunological ratings. 

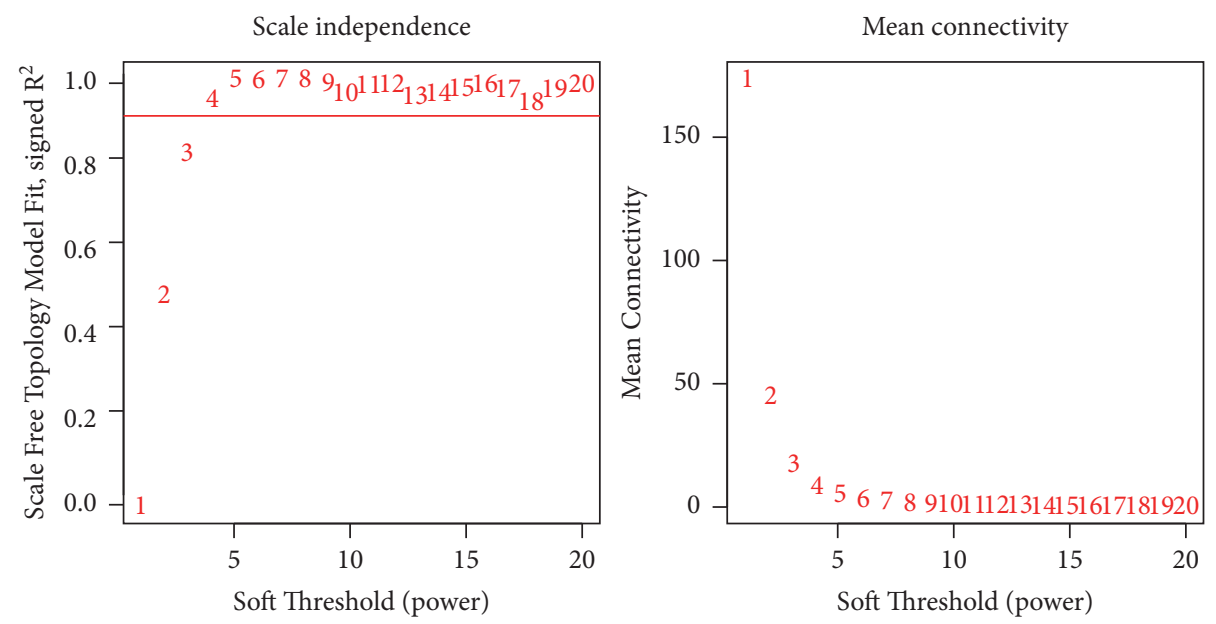

(a)

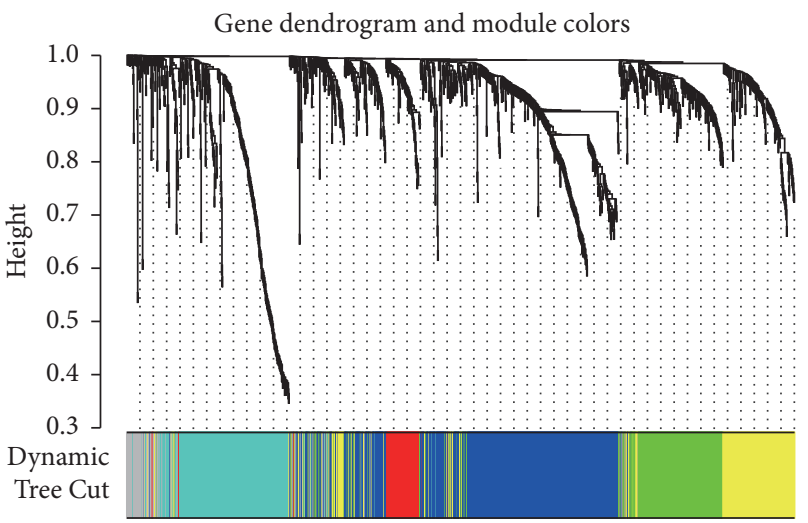

(b)

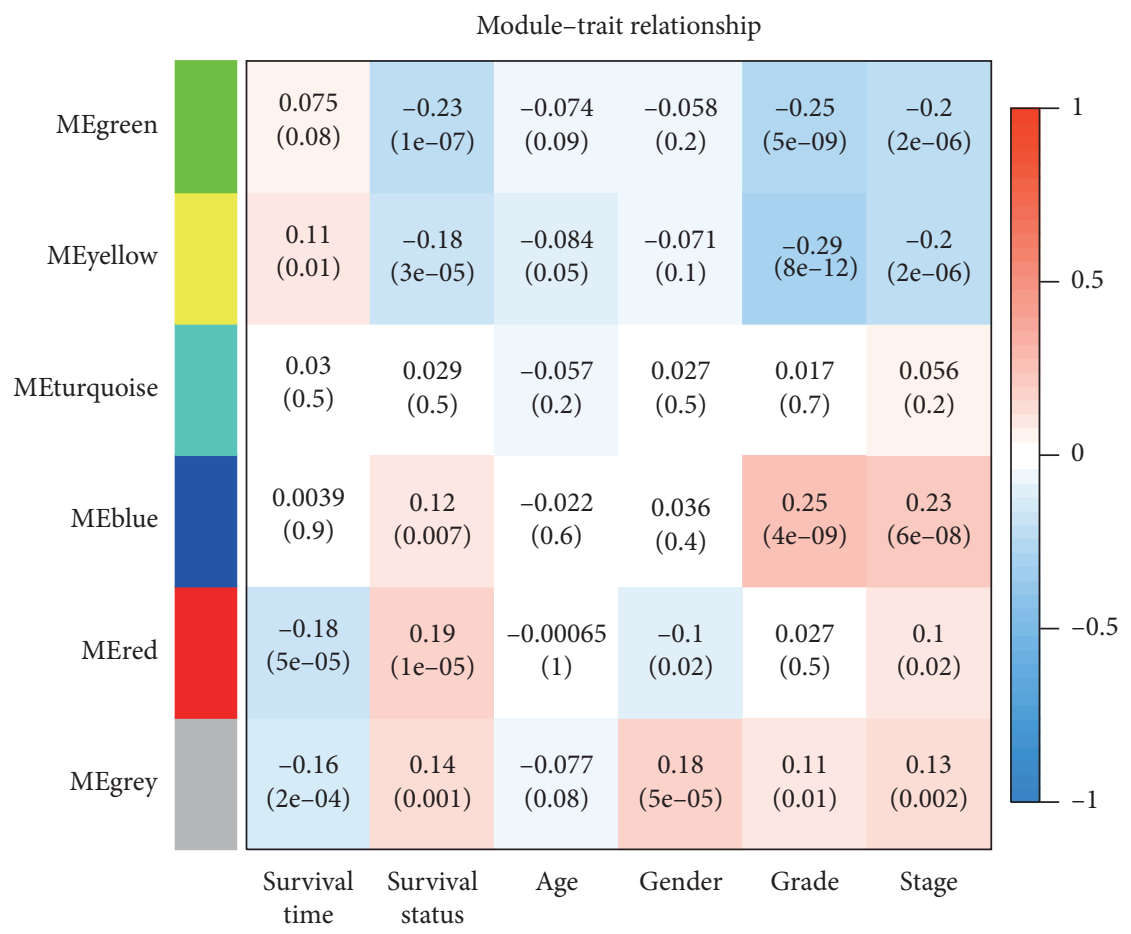

(c)

FIGURE 1: WGCNA network construction and module identification. (a) Soft thresholds determined. (b) WGCNA module identification and clustering dendrogram. (c) Matrix of eigengene values and clinical characteristics. 


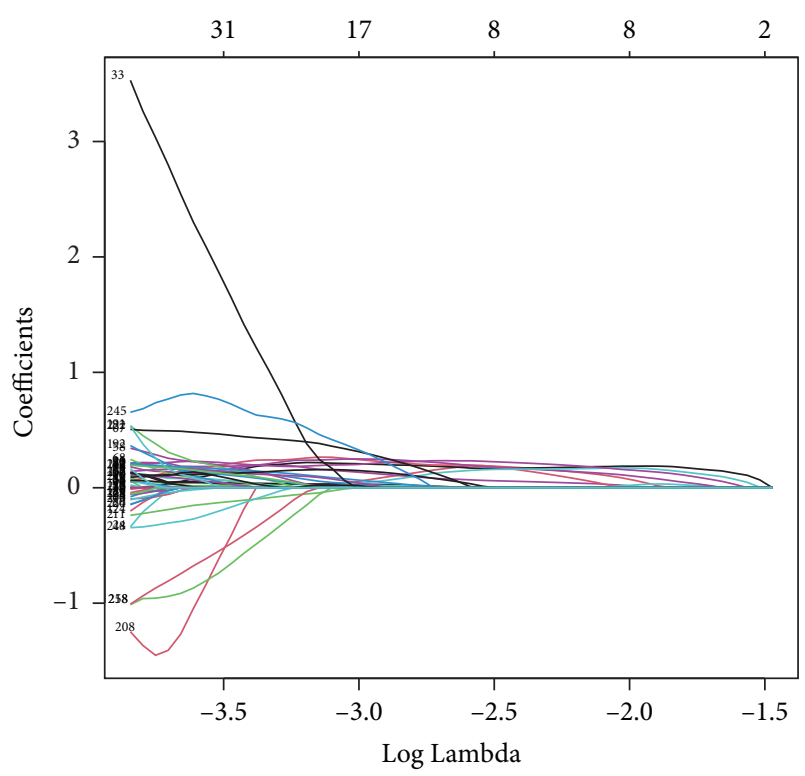

(a)

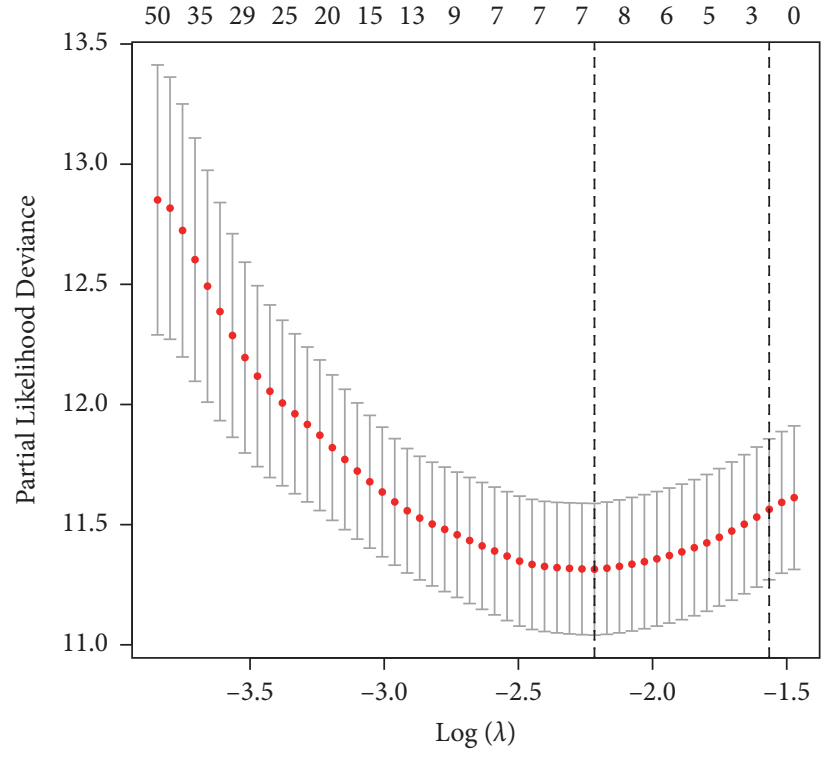

(b)

Figure 2: LASSO Cox regression identified $7 \operatorname{lncRNAs}$ for model construction $(a, b)$.

TABLE 2: IR-lncRNAs from the predictive model of TCGA are associated with OS.

\begin{tabular}{lcccc}
\hline Gene & HR & 95\% CI & $P$ value & LASSO coef. \\
\hline DLGAP1-AS2 & 3.749 & $2.860-4.912$ & $<0.001$ & 0.140099926 \\
AC024060.2 & 2.225 & $1.791-2.762$ & $<0.001$ & 0.041871909 \\
SLBP-DT & 2.628 & $2.057-3.355$ & $<0.001$ & 0.177728419 \\
DGUOK-AS1 & 3.115 & $2.187-4.435$ & $<0.001$ & 0.085301062 \\
MYG1-AS1 & 2.195 & $1.785-2.698$ & $<0.001$ & 0.141712499 \\
AC005785.1 & 2.821 & $2.127-3.740$ & $<0.001$ & 0.164170337 \\
MELTF-AS1 & 2.273 & $1.899-2.719$ & $<0.001$ & 0.192870454 \\
\hline
\end{tabular}

Furthermore, patients with high immunological ratings were linked to a worse prognosis (Figure 6(b)).

3.7. The Landscape of Immune Infiltration in KIRC. We show the content of 22 immune cells in each sample by bar plot, as shown in Figure 7(a). Heat map of 22 immune cells is shown in Figure 7(b). Furthermore, using the Wilcoxon rank-sum test, we discovered that CD8T cells, naïve B cells, follicular helper T cells, regulatory $\mathrm{T}$ cells (Tregs), M2Macrophages, and resting mast cells were substantially different between high- and low-risk individuals (Figure 7(c)). Furthermore, $\mathrm{K}-\mathrm{M}$ analysis indicated that patients with low proportions of resting dendritic and mast cells had a poor prognosis. $\mathrm{Pa}$ tients with a high number of follicular helper and regulatory T cells (Tregs) had a better prognosis (Figures $7(\mathrm{~d})-7(\mathrm{~g})$ ).

3.8. GSEA. We used GSEA analysis on the high- and lowrisk groups to further study the essential signalling pathways. Our results showed that alpha linolenic acid metabolism, arachidonic acid metabolism, homologous recombination, glycerophospholipid metabolism, linoleic acid metabolism, and ether lipid metabolism were enriched (Figure 8).
3.9. Screening for DEGs. We found 765 DEGs between highand low-risk groups, comprising 6 downregulated and 759 upregulated genes (Figure S1). Based on these DEGs, we ran a pathway enrichment analysis. They were primarily enriched in immune-related pathways, such as $\mathrm{T}$ cell proliferation regulation and $\mathrm{T}$ cell activation (Figure 9).

3.10. Potential Small Molecule Drugs. We uploaded 765 DEGs with $|\log 2 \mathrm{FC}| \geq 1$ to the CMap website. Among these highly correlated molecules, tanespimycin, ursodeoxycholic acid, and helveticoside had the highest degree of negative correlation with patients in the high-risk group of KIRC (Figure 10). All of them may have a potential therapeutic effect on KIRC patients in the high-risk group.

3.11. Crosstalk with Acute Kidney Injury. The incidence of tumors has increased and so has the number of patients with kidney injury caused by tumors. Acute kidney injury is a common complication of the cancer itself or the treatment process. To find the relationship between high- and low-risk groups of KIRC patients and AKI, we downloaded GSE139061, which contains 39 native human kidney biopsy samples (AKI group) and 9 reference nephrectomy samples (REF group); we found 44 shared DEGs (Figure S2). Future studies are needed to elucidate the relationship between KIRC and AKI.

\section{Discussion}

Kidney cancer has been on the rise in terms of incidence and death in recent years, and its treatment remains a serious issue globally, owing to its dismal prognosis [23]. As a result, identifying patients with a bad prognosis is a critical task. Despite the findings of various prognostic studies, TNM 


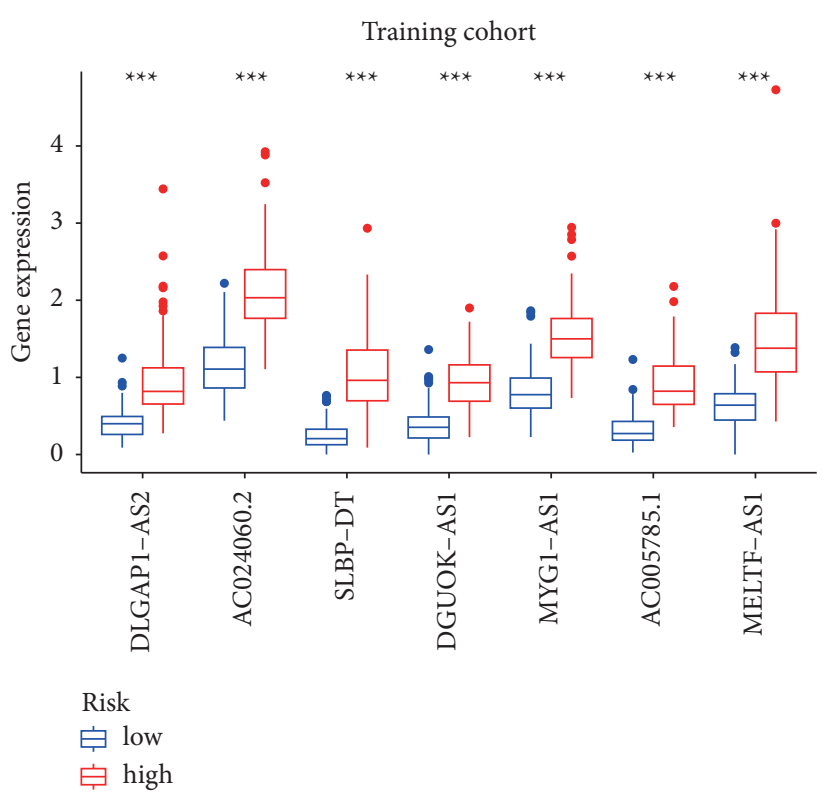

(a)

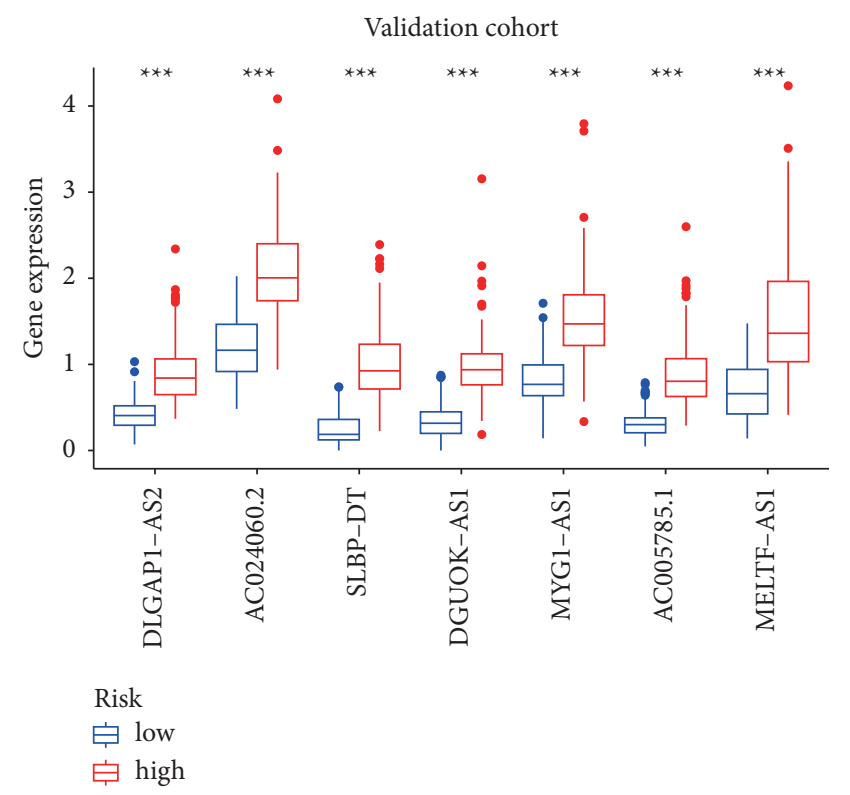

(b)

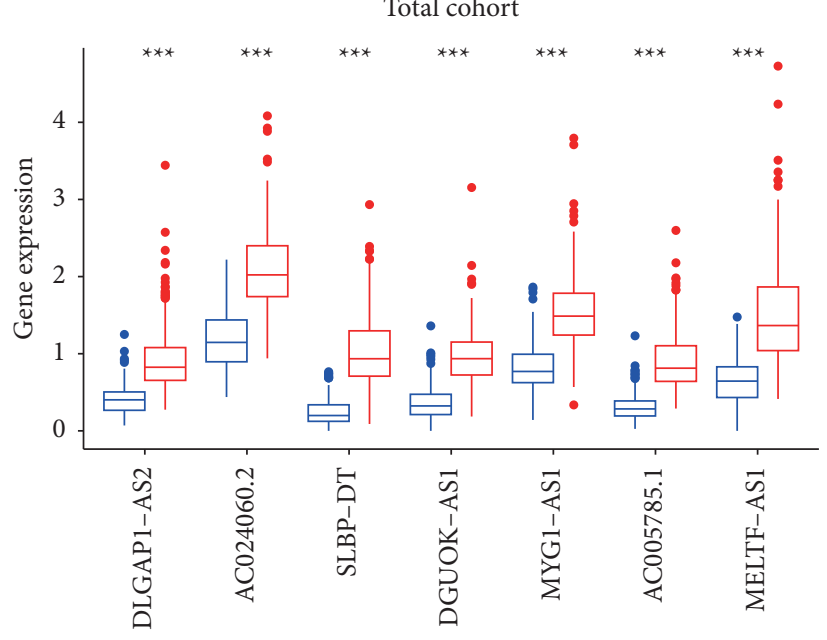

Risk

追 low

追 high

(c)

FIGURE 3: Expression levels of seven biomarkers in the training group (a), validation group (b), and total cohort (c) in the high-risk and lowrisk group cohorts.

staging remains the most accurate predictor of KIRC [24]. However, because of the variability of KIRC, clinical outcomes among individuals with the same TNM stage may differ dramatically [25]. As a result, finding reliable prognostic biomarkers is critical for building an accurate prognosis model. Because of the role of the immune system in cancer growth and the existence of a unique immunological milieu in the kidney, the search for immune-related biomarkers is essential for the prognosis of KIRC patients and may help guide immunotherapy research.

We used IRG and IR-lncRNAs to create the first predictive model for KIRC patients in this research. We built and validated the predictive model based on seven IR-
lncRNAs. The findings revealed that the predictive model correctly categorized KIRC patients into high- and low-risk groups, with substantial variations in OS between the risk groups. This predictive model's prognostic value was also verified, demonstrating that the predictive model based on IRG and IR-lncRNAs is reliable and valuable.

These seven IR-lncRNA indicators all showed a good correlation with prognosis. Other biomarkers utilized in our prognosis model have been reported by research, even though certain IR-lncRNAs in our predictive model have not been functionally annotated and elucidated. The lncRNA DLGAP1-AS1 has been demonstrated to increase Wnt1 transcription and gastric cancer growth by interacting with 
TABLE 3: Correlation between the training cohort, validation cohort, and total cohort clinical features and the risk scores of the predictive model based on immune-related lncRNAs.

\begin{tabular}{|c|c|c|c|c|c|c|c|c|c|c|c|c|}
\hline \multirow[b]{2}{*}{ Variables } & \multicolumn{4}{|c|}{ Training cohort $(n=264)$} & \multicolumn{4}{|c|}{ Validation cohort $(n=262)$} & \multicolumn{4}{|c|}{ Total cohort $(n=536)$} \\
\hline & $\begin{array}{l}\text { High risk } \\
(\%)\end{array}$ & $\begin{array}{c}\text { Low risk } \\
(\%)\end{array}$ & $\chi^{2}$ & $P$ & $\begin{array}{l}\text { High risk } \\
(\%)\end{array}$ & $\begin{array}{c}\text { Low risk } \\
(\%)\end{array}$ & $x^{2}$ & $P$ & $\begin{array}{l}\text { High risk } \\
(\%)\end{array}$ & $\begin{array}{c}\text { Low risk } \\
(\%)\end{array}$ & $\chi^{2}$ & $P$ \\
\hline \multicolumn{13}{|l|}{ Age } \\
\hline$\leq 65$ & $\begin{array}{c}86 \\
(65.15 \%)\end{array}$ & $\begin{array}{c}88 \\
(66.67 \%)\end{array}$ & 0.017 & 0.897 & $\begin{array}{c}86 \\
(65.65 \%)\end{array}$ & $\begin{array}{c}87 \\
(66.41 \%)\end{array}$ & 0 & 1 & $\begin{array}{c}172 \\
(65.4 \%)\end{array}$ & $\begin{array}{c}175 \\
(66.54 \%)\end{array}$ & 0.034 & 0.854 \\
\hline$>65$ & $\begin{array}{c}46 \\
(34.85 \%)\end{array}$ & $\begin{array}{c}44 \\
(33.33 \%)\end{array}$ & & & $\begin{array}{c}45 \\
(34.35 \%)\end{array}$ & $\begin{array}{c}44 \\
(33.59 \%)\end{array}$ & & & $91(34.6 \%)$ & $\begin{array}{c}88 \\
(33.46 \%)\end{array}$ & & \\
\hline \multicolumn{13}{|l|}{ Gender } \\
\hline Female & $\begin{array}{c}44 \\
(33.33 \%)\end{array}$ & $\begin{array}{c}44 \\
(33.33 \%)\end{array}$ & 0 & 1 & $\begin{array}{c}47 \\
(35.88 \%)\end{array}$ & $\begin{array}{c}48 \\
(36.64 \%)\end{array}$ & 0 & 1 & $91(34.6 \%)$ & $\begin{array}{c}92 \\
(34.98 \%)\end{array}$ & 0 & 1 \\
\hline Male & $\begin{array}{c}88 \\
(66.67 \%)\end{array}$ & $\begin{array}{c}88 \\
(66.67 \%)\end{array}$ & & & $\begin{array}{c}84 \\
(64.12 \%)\end{array}$ & $\begin{array}{c}83 \\
(63.36 \%)\end{array}$ & & & $\begin{array}{c}172 \\
(65.4 \%)\end{array}$ & $\begin{array}{c}171 \\
(65.02 \%)\end{array}$ & & \\
\hline \multicolumn{13}{|l|}{ Grade } \\
\hline G1-2 & $\begin{array}{c}54 \\
(40.91 \%)\end{array}$ & $\begin{array}{c}62 \\
(46.97 \%)\end{array}$ & & & $\begin{array}{c}56 \\
(42.75 \%)\end{array}$ & $\begin{array}{c}67 \\
(51.15 \%)\end{array}$ & & & $\begin{array}{c}110 \\
(41.83 \%)\end{array}$ & $\begin{array}{c}129 \\
(49.05 \%)\end{array}$ & & \\
\hline G3-4 & $\begin{array}{c}78 \\
(59.09 \%)\end{array}$ & $\begin{array}{c}68 \\
(51.52 \%)\end{array}$ & 0.962 & 0.327 & $\begin{array}{c}73 \\
(55.73 \%)\end{array}$ & $60(45.8 \%)$ & 1.880 & 0.170 & $\begin{array}{c}151 \\
(57.41 \%)\end{array}$ & $\begin{array}{c}128 \\
(48.67 \%)\end{array}$ & 3.056 & 0.08 \\
\hline $\begin{array}{l}\text { Unknown } \\
\text { Stage }\end{array}$ & $0(0 \%)$ & $2(1.52 \%)$ & & & $2(1.53 \%)$ & $4(3.05 \%)$ & & & $2(0.76 \%)$ & $6(2.28 \%)$ & & \\
\hline Stages I-II & $\begin{array}{c}67 \\
(50.76 \%)\end{array}$ & $\begin{array}{c}91 \\
(68.94 \%)\end{array}$ & & & $\begin{array}{c}76 \\
(58.02 \%)\end{array}$ & $\begin{array}{c}84 \\
(64.12 \%)\end{array}$ & & & $\begin{array}{c}143 \\
(54.37 \%)\end{array}$ & $\begin{array}{c}175 \\
(66.54 \%)\end{array}$ & & \\
\hline Stages III-IV & $\begin{array}{c}65 \\
(49.24 \%)\end{array}$ & $\begin{array}{c}41 \\
(31.06 \%)\end{array}$ & 8.339 & 0.004 & $\begin{array}{c}53 \\
(40.46 \%)\end{array}$ & $\begin{array}{c}46 \\
(35.11 \%)\end{array}$ & 0.666 & 0.414 & $\begin{array}{c}118 \\
(44.87 \%)\end{array}$ & $\begin{array}{c}87 \\
(33.08 \%)\end{array}$ & 7.41 & 0.006 \\
\hline $\begin{array}{l}\text { Unknown } \\
\mathrm{T} \\
\text { classification }\end{array}$ & 1 & 1 & & & $2(1.53 \%)$ & $1(0.76 \%)$ & & & $2(0.76 \%)$ & $1(0.38 \%)$ & & \\
\hline $\mathrm{T} 1-2$ & $\begin{array}{c}72 \\
(54.55 \%)\end{array}$ & $\begin{array}{c}91 \\
(68.94 \%)\end{array}$ & 5.196 & 0.023 & $\begin{array}{c}80 \\
(61.07 \%)\end{array}$ & $\begin{array}{c}93 \\
(70.99 \%)\end{array}$ & 2.450 & 0.117 & $\begin{array}{c}152 \\
(57.79 \%)\end{array}$ & $\begin{array}{c}184 \\
(69.96 \%)\end{array}$ & 7.918 & 0.005 \\
\hline T3-4 & $\begin{array}{c}60 \\
(45.45 \%)\end{array}$ & $\begin{array}{c}41 \\
(31.06 \%)\end{array}$ & & & $\begin{array}{c}51 \\
(38.93 \%)\end{array}$ & $\begin{array}{c}38 \\
(29.01 \%)\end{array}$ & & & $\begin{array}{c}111 \\
(42.21 \%)\end{array}$ & $\begin{array}{c}79 \\
(30.04 \%)\end{array}$ & & \\
\hline \multicolumn{13}{|l|}{$\begin{array}{l}\text { M } \\
\text { classification }\end{array}$} \\
\hline M0 & $94(71.21 \%)$ & $\begin{array}{c}119 \\
(90.15 \%)\end{array}$ & & & $\begin{array}{c}97 \\
(74.05 \%)\end{array}$ & $\begin{array}{c}108 \\
(82.44 \%)\end{array}$ & & & $\begin{array}{c}191 \\
(72.62 \%)\end{array}$ & $\begin{array}{c}227 \\
(86.31 \%)\end{array}$ & & \\
\hline M1 & $28(21.21 \%)$ & $9(6.82 \%)$ & 11.324 & $<0.0$ & $\begin{array}{c}20 \\
(15.27 \%)\end{array}$ & $\begin{array}{c}21 \\
(16.03 \%)\end{array}$ & 0 & 1 & $\begin{array}{c}48 \\
(18.25 \%)\end{array}$ & $\begin{array}{c}30 \\
(11.41 \%)\end{array}$ & 5.99 & .014 \\
\hline Unknown & $10(7.58 \%)$ & $4(3.03 \%)$ & & & $\begin{array}{c}14 \\
(10.69 \%)\end{array}$ & $2(1.53 \%)$ & & & $24(9.13 \%)$ & $6(2.28 \%)$ & & \\
\hline \multicolumn{13}{|l|}{$\begin{array}{l}\mathrm{N} \\
\text { classification }\end{array}$} \\
\hline No & $61(46.21 \%)$ & $59(44.7 \%)$ & 1.292 & 0.256 & $\begin{array}{c}61 \\
(46.56 \%)\end{array}$ & $\begin{array}{c}57 \\
(43.51 \%)\end{array}$ & 0.145 & 0.703 & $\begin{array}{c}122 \\
(46.39 \%)\end{array}$ & $\begin{array}{c}116 \\
(44.11 \%)\end{array}$ & 0.016 & 0.898 \\
\hline N1 & $5(3.79 \%)$ & $1(0.76 \%)$ & & & $4(3.05 \%)$ & $6(4.58 \%)$ & & & $9(3.42 \%)$ & $7(2.66 \%)$ & & \\
\hline Unknown & $66(50 \%)$ & $\begin{array}{c}72 \\
(54.55 \%)\end{array}$ & & & $\begin{array}{c}66 \\
(50.38 \%)\end{array}$ & $\begin{array}{c}68 \\
(51.91 \%)\end{array}$ & & & $\begin{array}{c}132 \\
(50.19 \%)\end{array}$ & $\begin{array}{c}140 \\
(53.23 \%)\end{array}$ & & \\
\hline
\end{tabular}

Six3 [26]. In breast cancer, DGUOK-AS1 may operate as a tumor promoter via modulating the miR-204-5p/IL-11 axis [27]. By modulating MMP14 expression, the lncRNA MELTF-AS1 may enhance osteosarcoma metastasis [28].

Immune cell infiltration of cancers has lately been associated with prognosis in many studies $[29,30]$. The ESTIMATE algorithm analyzes the particular gene expression profile of immune cells and generates an immune score to forecast immune cell infiltration. Immune cell infiltration correlates with patient prognosis in many tumors, according to several prior ESTIMATE assessments [31]. We discovered that risk scores based on predictive models were favourably connected with immune scores in the current investigation. Patients with high immunological ratings also had a worse prognosis. To learn more about immune cell infiltration, we ran another CIBERSORT study to look at the different kinds of invading cells. CD8 T cells, regulatory T cells (Tregs), and follicular helper T cells were shown to be more prevalent in the high-risk group. Regulatory T cells (Tregs) and follicular helper T cells have also been linked to a poor prognosis. The follicular helper $\mathrm{T}$ cells may play a significant part in the progression of KIRC, which should be looked into more in the future. We found through GSEA analysis that alpha linolenic acid and arachidonic acid that maintain cell 
Training cohort

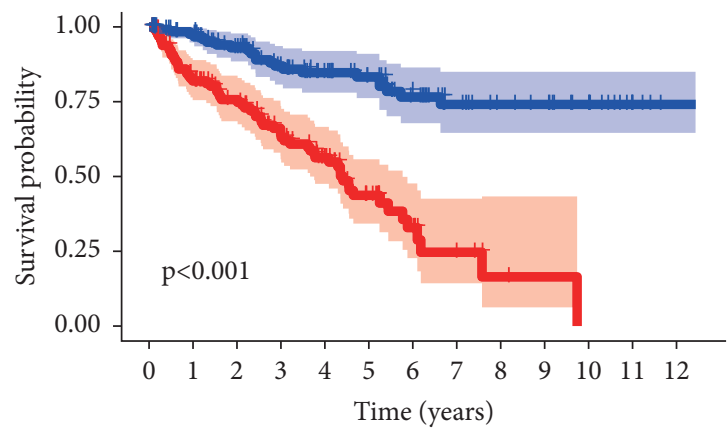

High risk - $\begin{array}{lllllllllllll}132 & 101 & 83 & 62 & 40 & 24 & 9 & 5 & 2 & 1 & 0 & 0 & 0\end{array}$ $\approx$ Low risk \begin{tabular}{c|cccccccccccc}
132 & 114 & 97 & 82 & 67 & 56 & 40 & 27 & 22 & 19 & 11 & 3 & 1 \\
\hline 1 & 1 & 2 & 3 & 4 & 5 & 6 & 7 & 1 & 9 & 1 & 1 & 1
\end{tabular} Time (years)

Risk

High risk

눌 risk

(a)

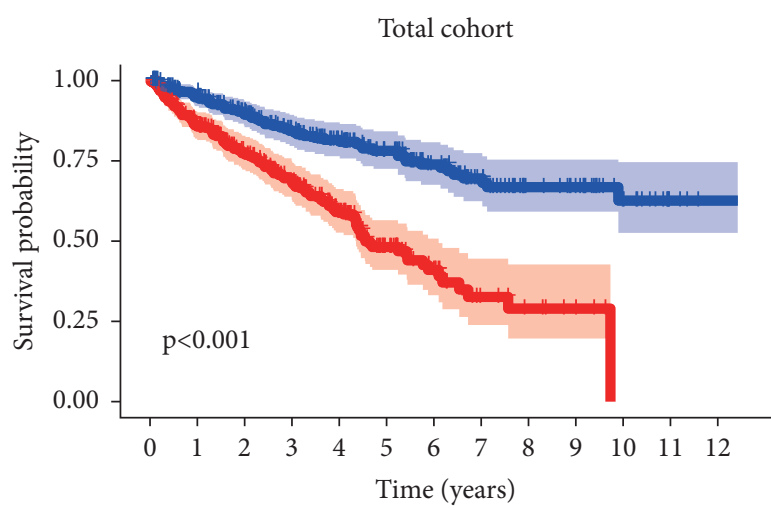

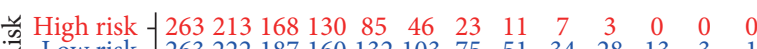
2. Low risk \begin{tabular}{|rrrrrrrrrrrrr}
263 & 222 & 187 & 160 & 132 & 103 & 75 & 51 & 34 & 28 & 13 & 3 & 1 \\
\hline 0 & 1 & 2 & 3 & 4 & 5 & 6 & 7 & 8 & 9 & 10 & 11 & 12
\end{tabular} Time (years)

Risk

- High risk

Low risk

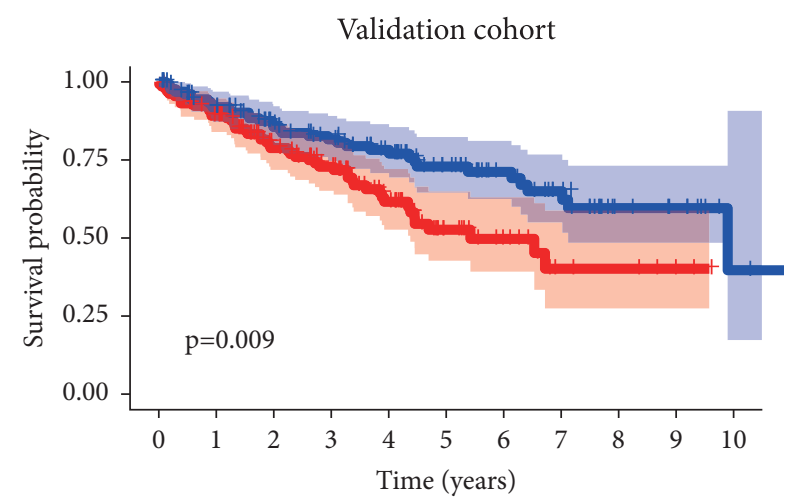

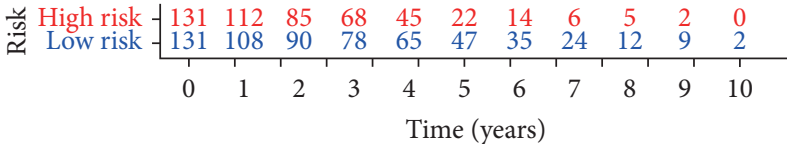

Risk

High risk

드 Low risk

(b)

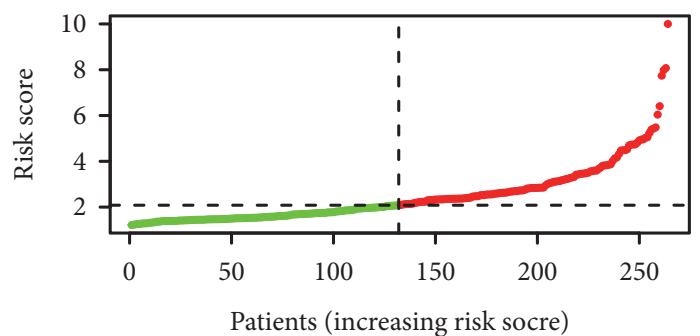

- High Risk

- Low Risk

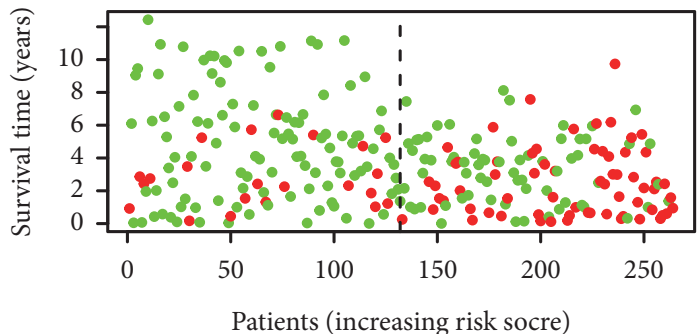

- Dead

- Aliv

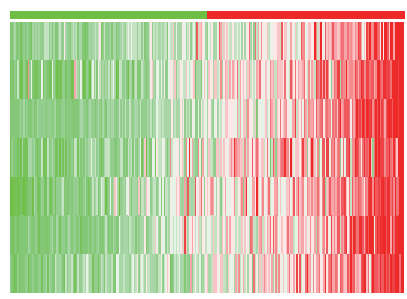
Risk $1-{ }^{6}$ Risk $\begin{array}{lll}\text { AC024060.2 } & 4 & \text { low } \\ \text { high }\end{array}$ SLBP-DT DGUOK-AS1 -2 MYG1-AS1 AC005785.1 MELTF-AS1 


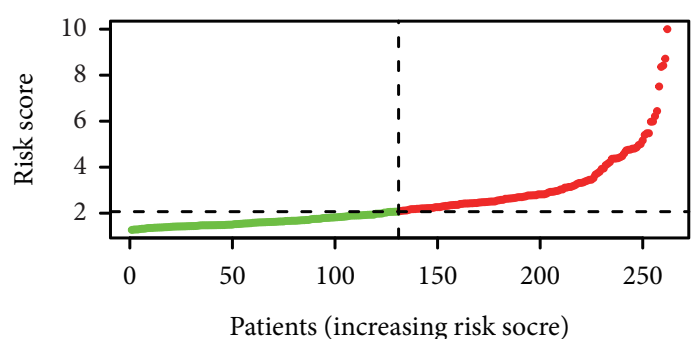

- High Risk

- Low Risk

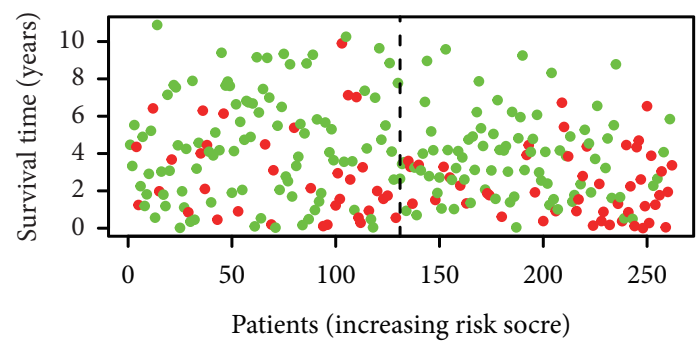

- Dead

- Aliv

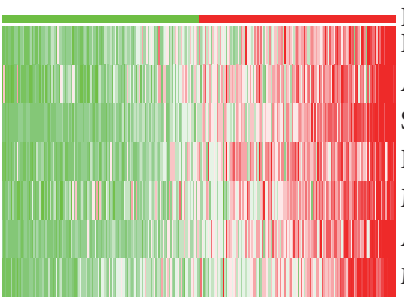

(e)

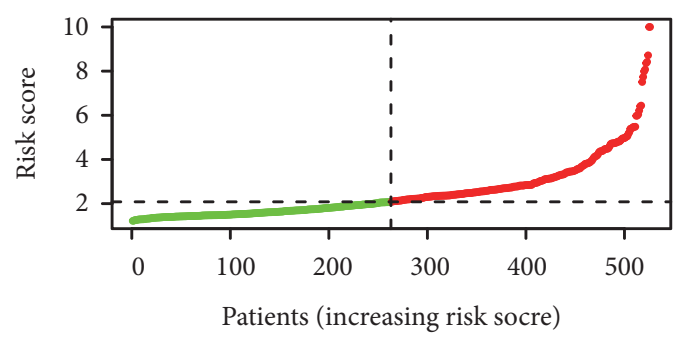

- High Risk

- Low Risk

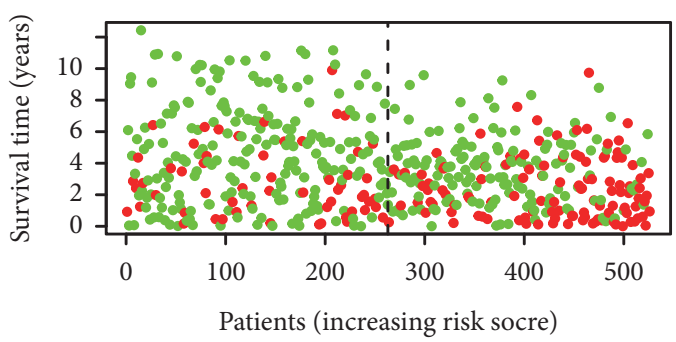

- Dead

- Aliv

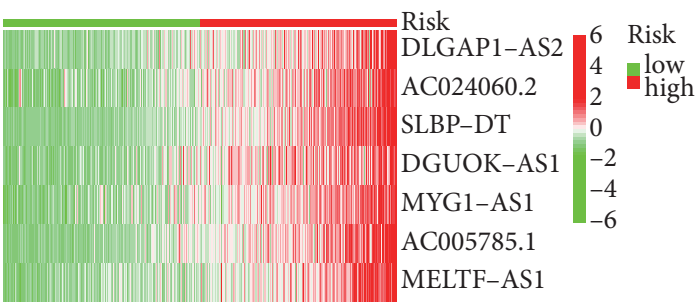

(f)

FIGURE 4: The predictive value of a model based on IR-lncRNAs. OS for high- and low-risk patients in the three cohorts (a-c) analyzed using the K-M method. Risk scores, survival status, and expression distribution of all seven lncRNAs in the prognostic model for patients in highand low-risk groups were calculated in the three cohorts (d-f).

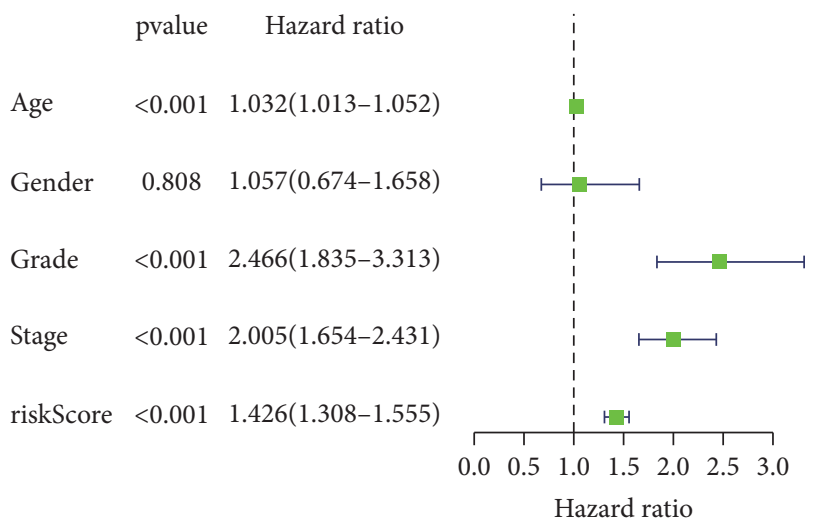

(a)

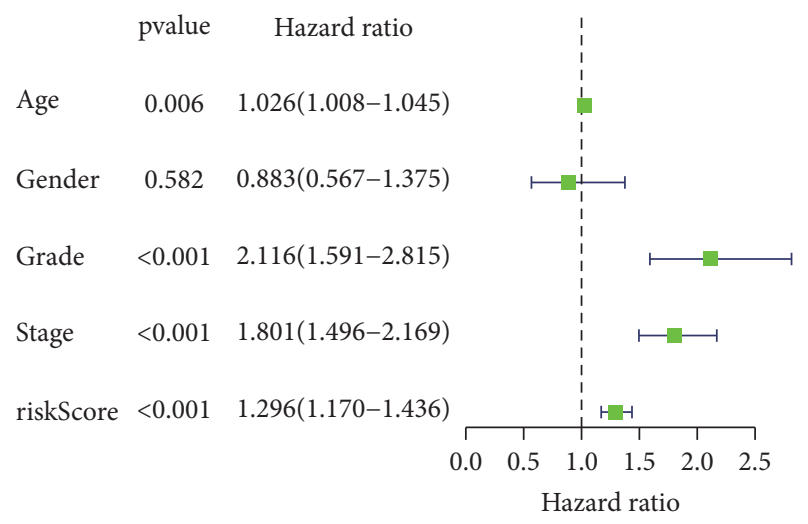

(b)

FIGURE 5: Continued. 


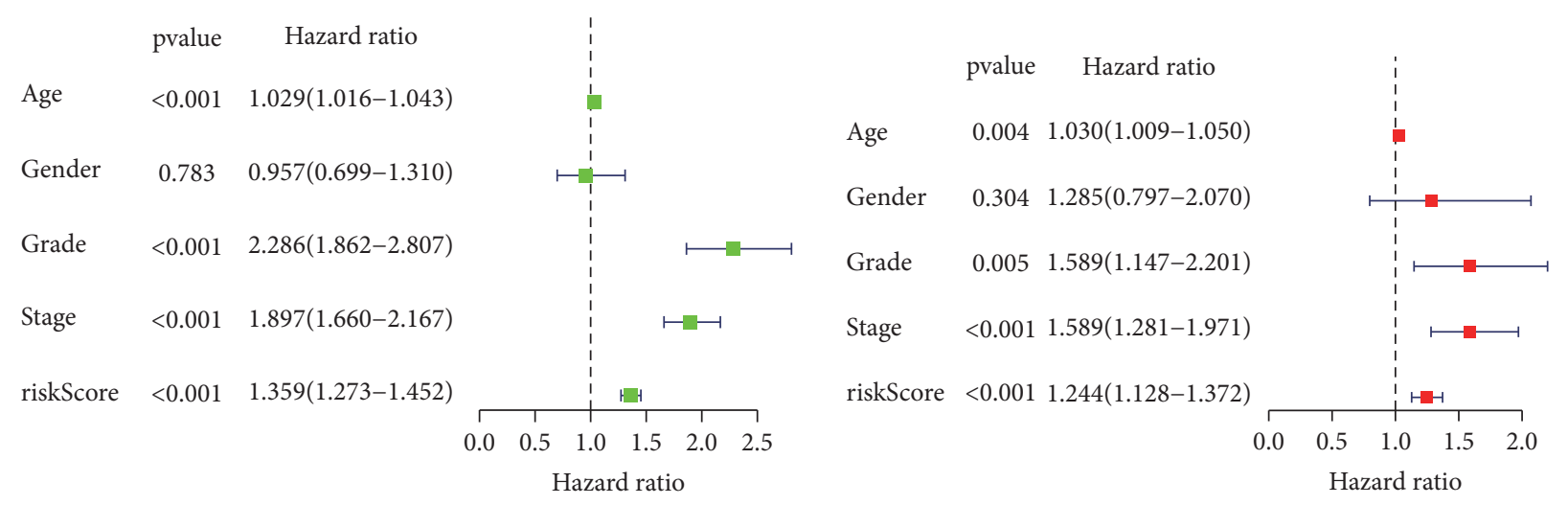

(c)

(d)

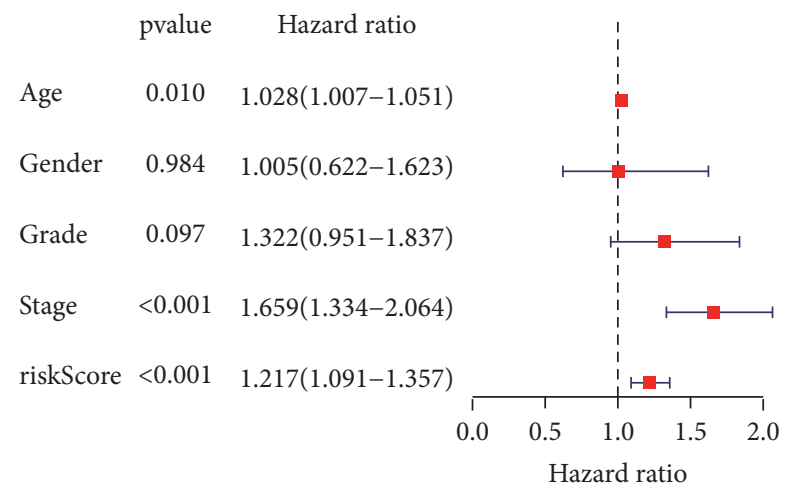

(e)

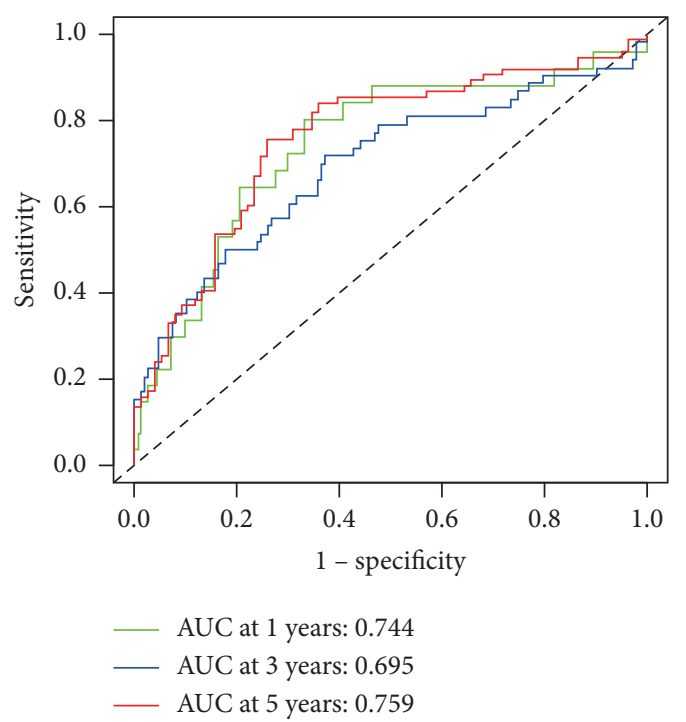

(g)

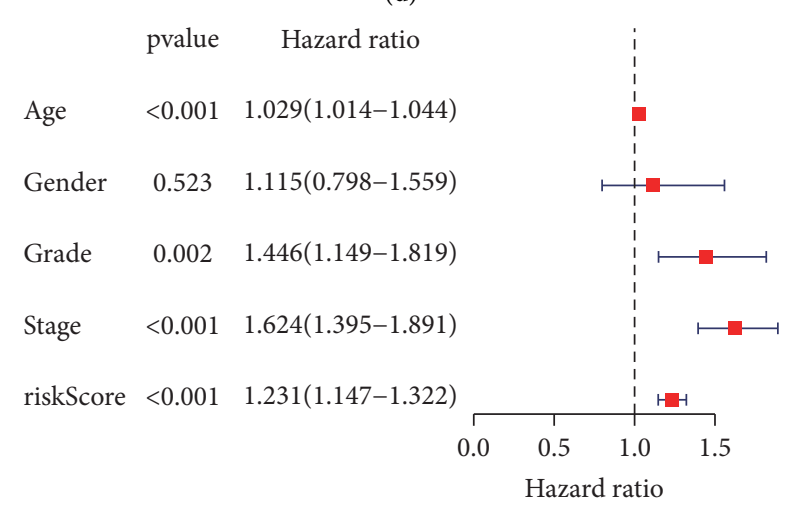

(f)

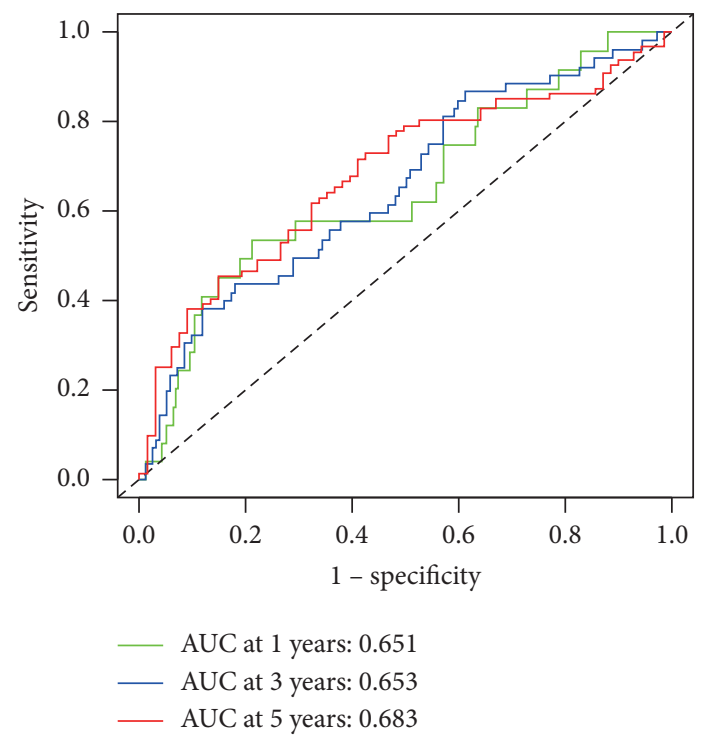

(h)

FIgURE 5: Continued. 


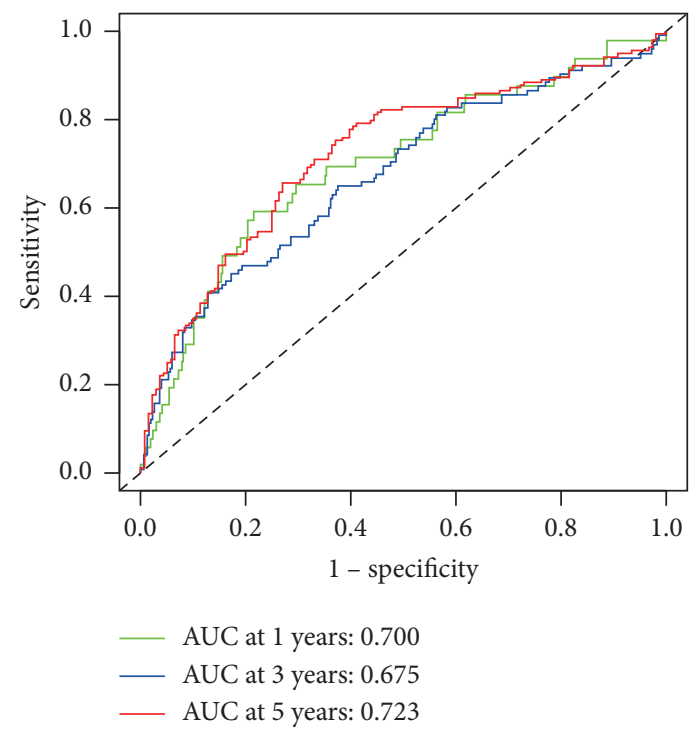

(i)

Figure 5: Prognostic value of the signature. Univariate and multivariate Cox analyses of predictive model in the training (a, $d$ ), validation $(b, e)$, and total cohorts (c, f). AUC for 1-, 3-, and 5-year OS prediction for the prognostic models in three cohorts (g-i).

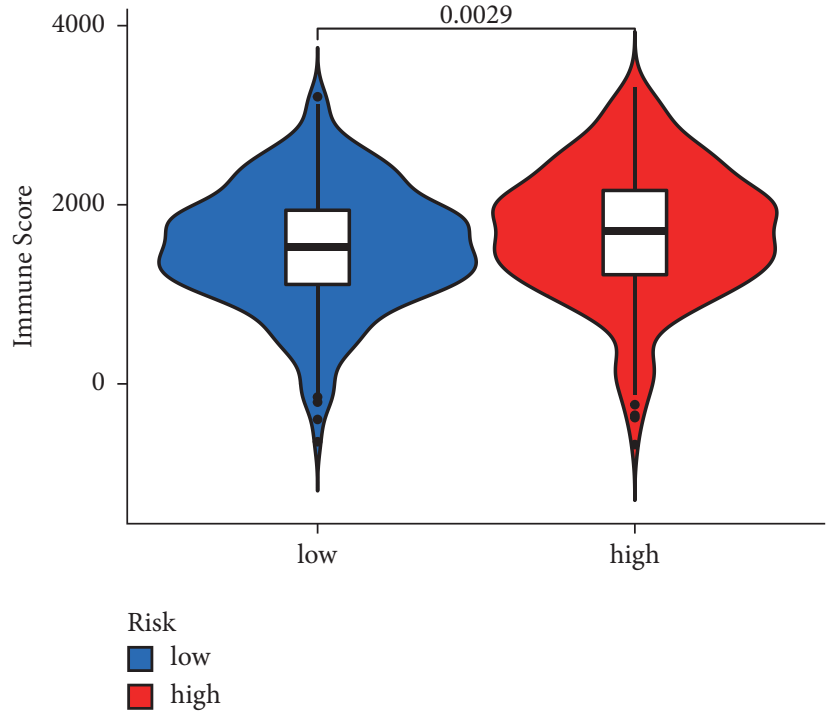

(a)

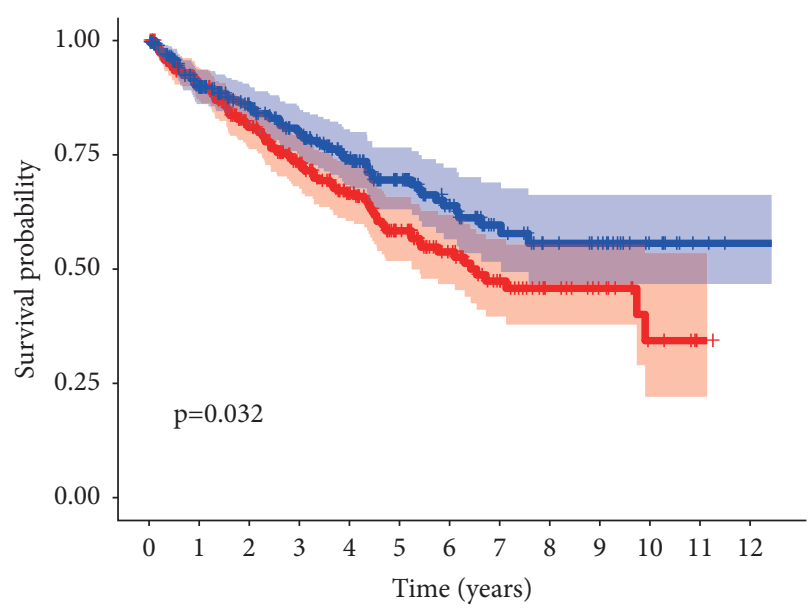

ImmuneScore
+ high
+ low

(b)

Figure 6: (a) High immune score in patients with high-risk score. (b) K-M analysis of OS between high and low immune scores.

structure and function also enhance immune functions and regulate lipid metabolism and related gene expression $[32,33]$.

Despite attempts by researchers to enhance KIRC patients' prognosis, the prognosis of KIRC patients has remained bad for decades. In this investigation, three small compounds, tanespimycin, ursodeoxycholic acid, and helveticoside, were discovered to have potential therapeutic benefits in individuals with KIRC. Tanespimycin is a geldanamycin derivative that has been studied for use in the treatment of cancer, notably in young individuals with specific forms of leukemia or solid tumors $[34,35]$. It is used to treat young kids with specific forms of leukemia or solid tumors, particularly kidney cancers. Helveticoside is a bioactive component of Descurainia sophia seed extract. Several investigations have shown this chemical to regulate genes involved in proliferation and apoptosis in caterpillar lung carcinoma cells [36]. Ursodeoxycholic acid is a secondary bile acid that is formed by intestinal bacteria and is necessary for lipid metabolism as well as the integrity of the intestinal barrier. Ursodeoxycholic acid has also been studied for its impact on tumor cell migration, tumor stem cells, and drug-induced dysbiosis [37, 38]. Ursodeoxycholic acid may also protect against the effects of cancer-fighting chemotherapeutics. 


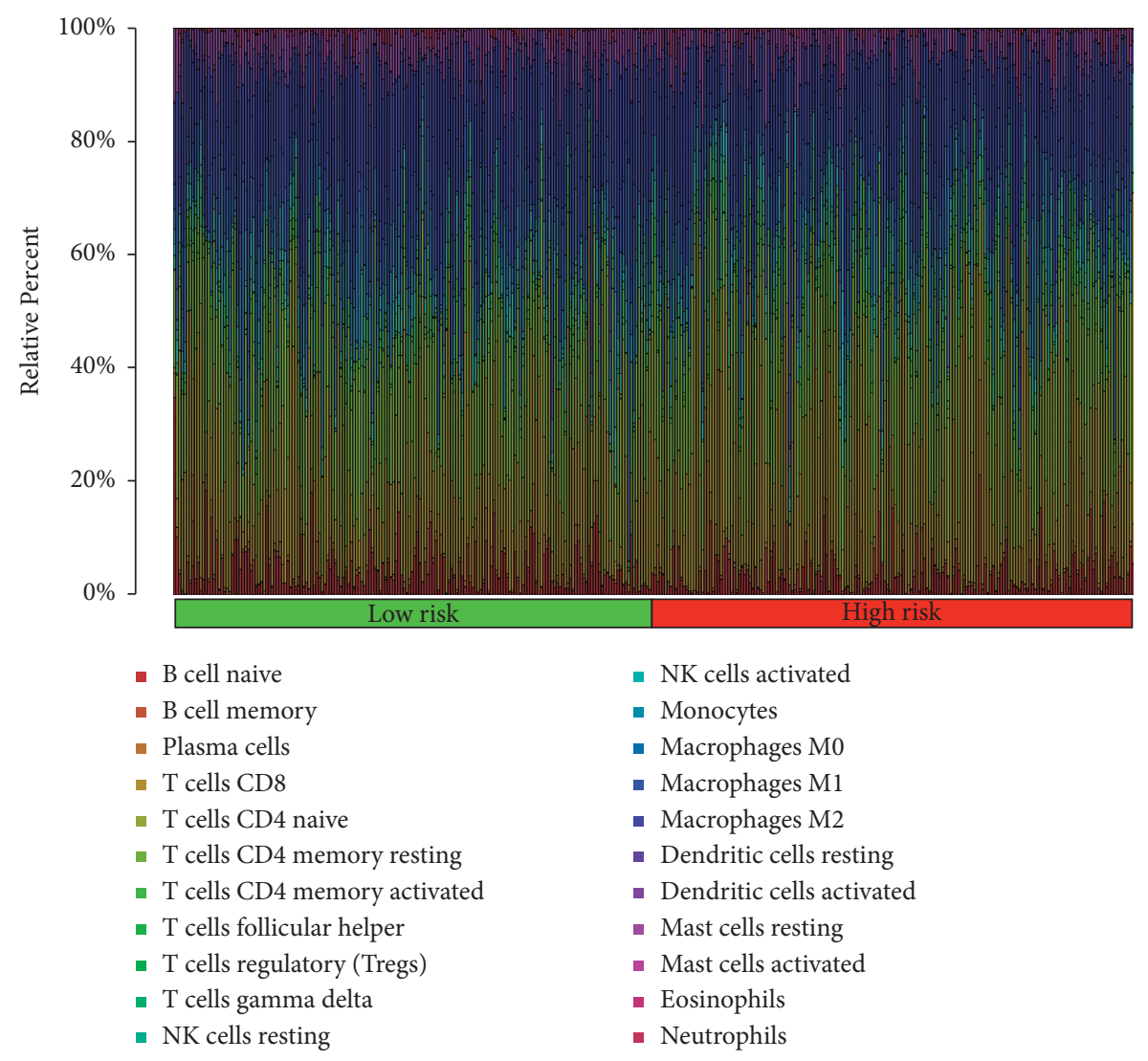

(a)

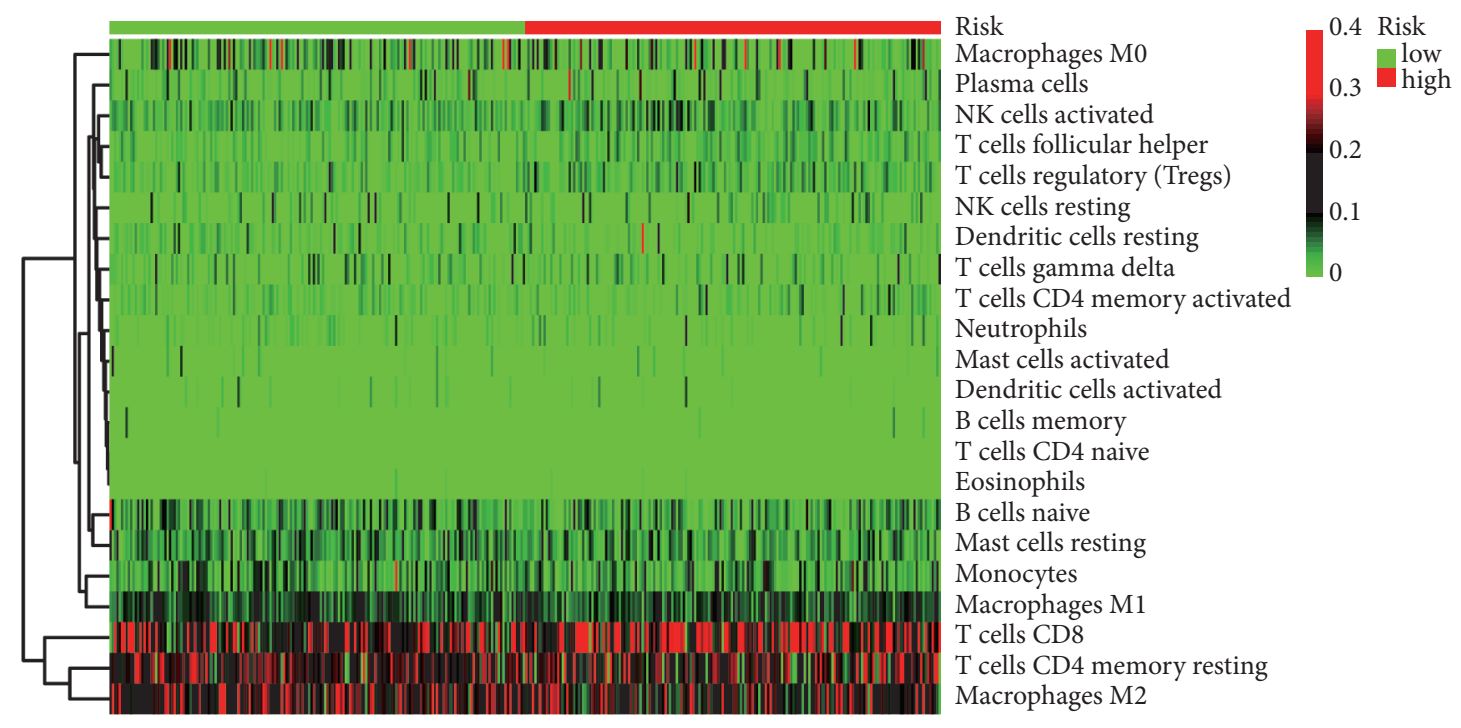

(b)

Figure 7: Continued. 


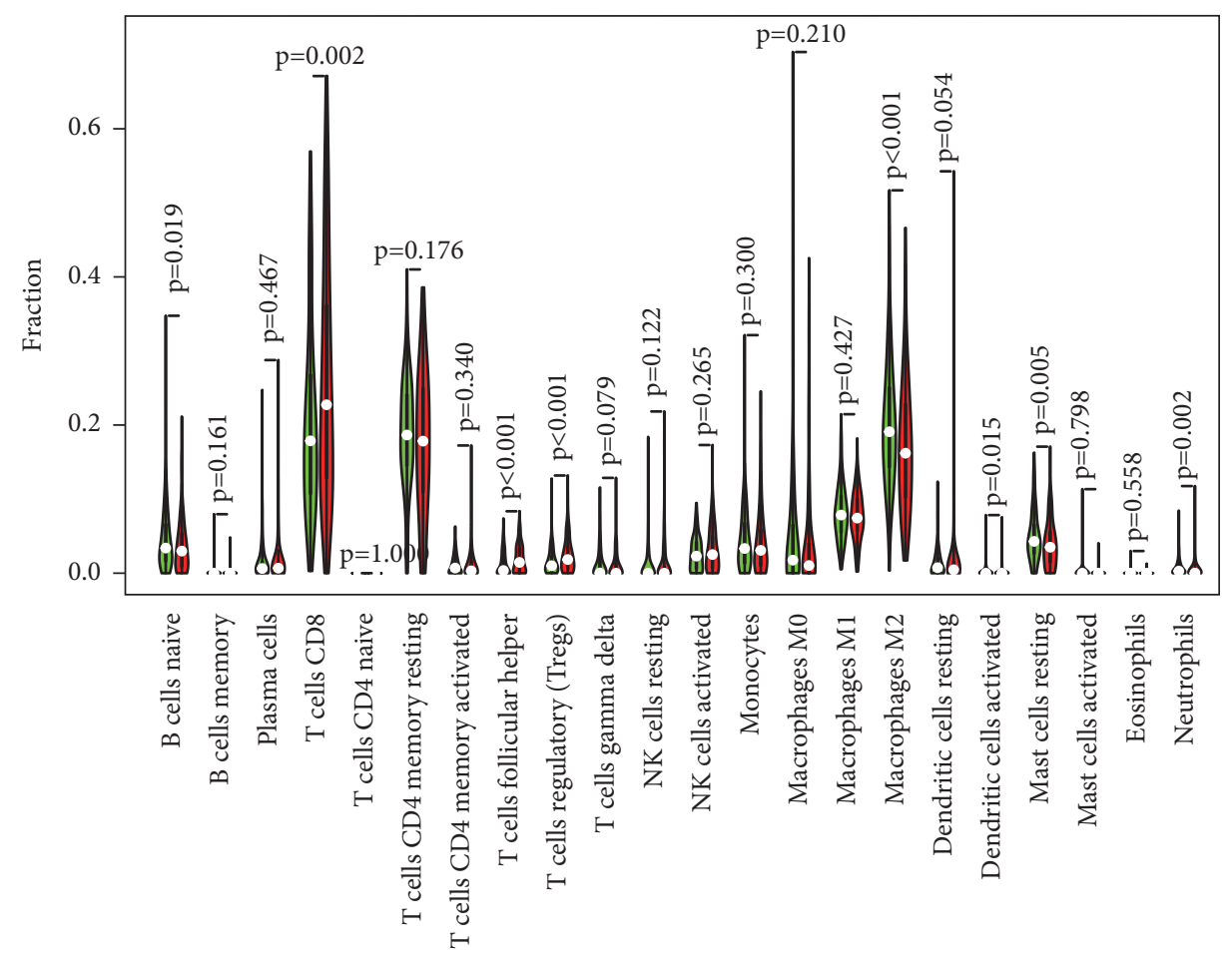

_ Low risk

— High risk

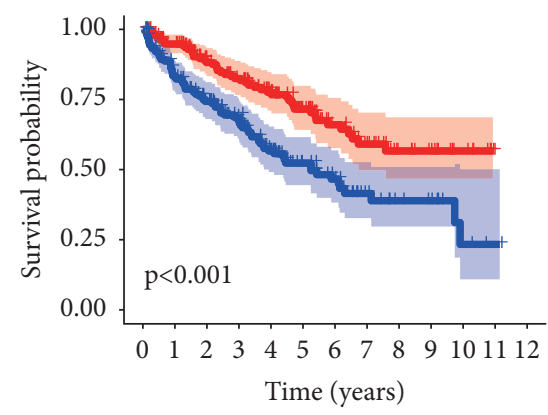

(c)

Dendritic cells resting

$\div$ high

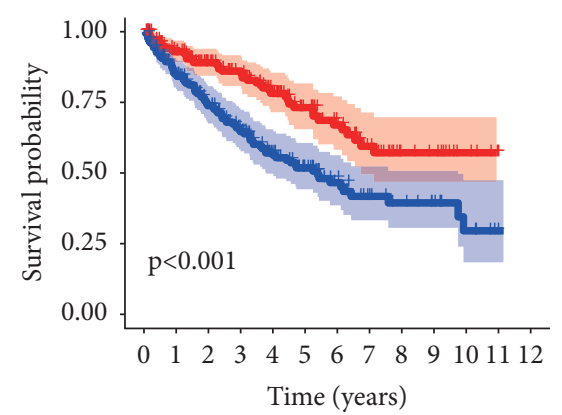

Mast cells resting

+ high

low

(d)

(e)

Figure 7: Continued. 


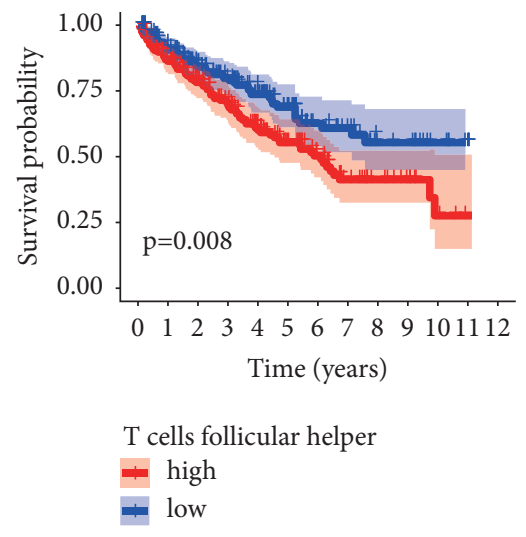

(f)

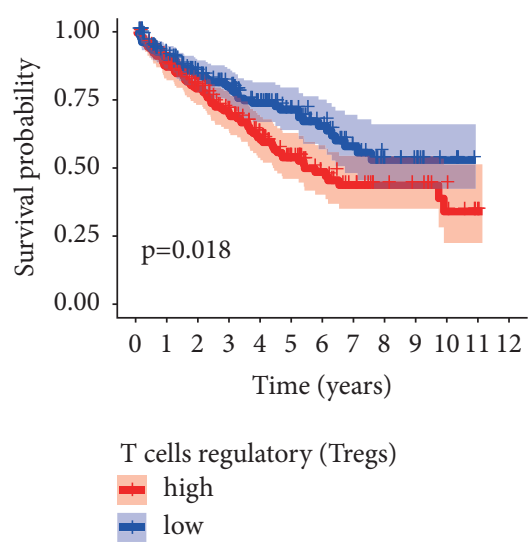

(g)

FIGURE 7: Composition of immune cells (a) and heat map (b) calculated by the CIBERSORT algorithm for each sample. (c) Differences in immune cell content between high- and low-risk groups. $(\mathrm{d}-\mathrm{g})$ Kaplan-Meier analysis of OS between high and low levels of the four immune cells.
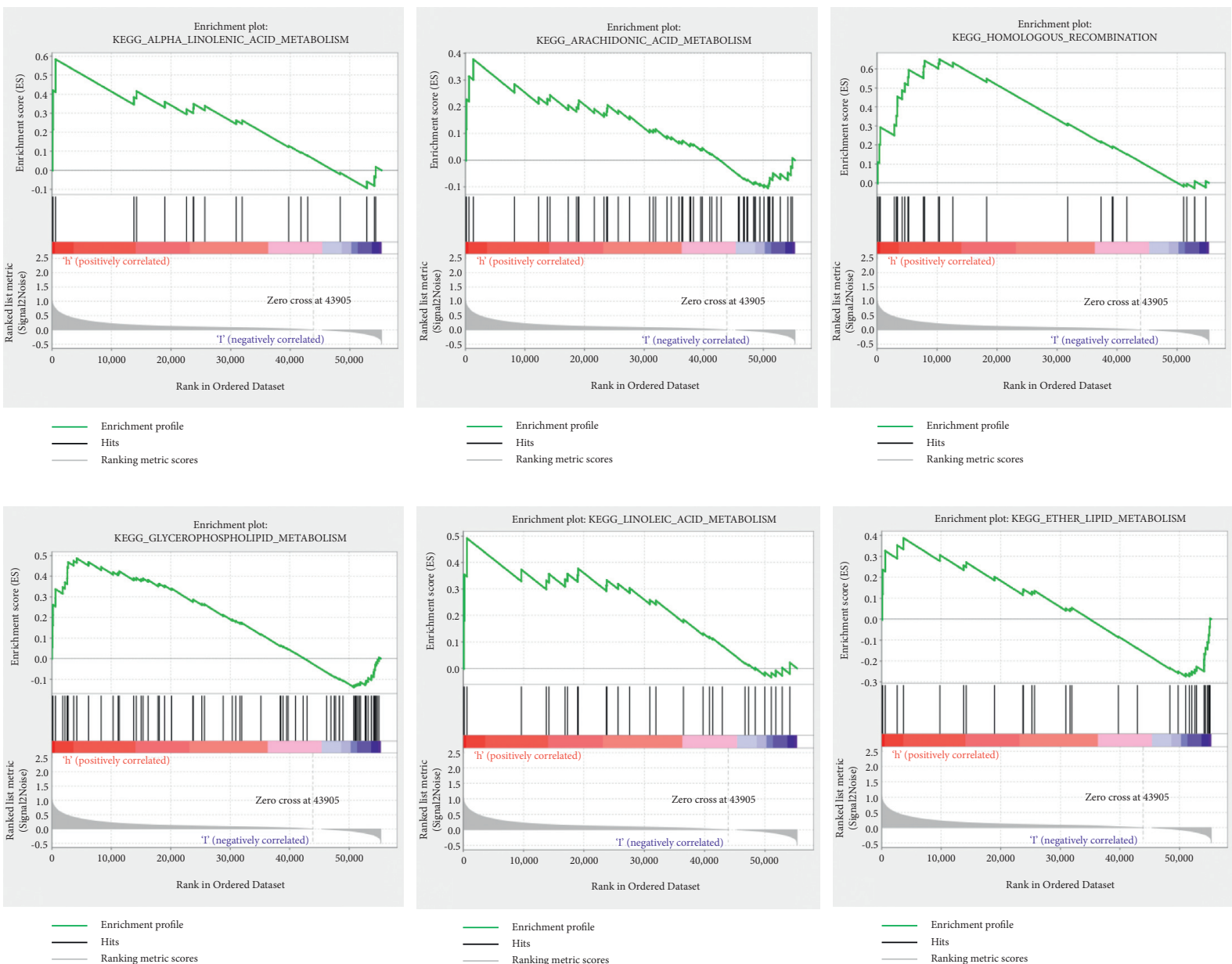

FIGURE 8: GSEA enrichment between the two groups.

This study has some limitations. First, it is based on one public database. Second, there were no cohorts from other databases for validation. Therefore, a large multicenter study is needed to confirm our findings before our predictive model can be applied to the clinicians. 


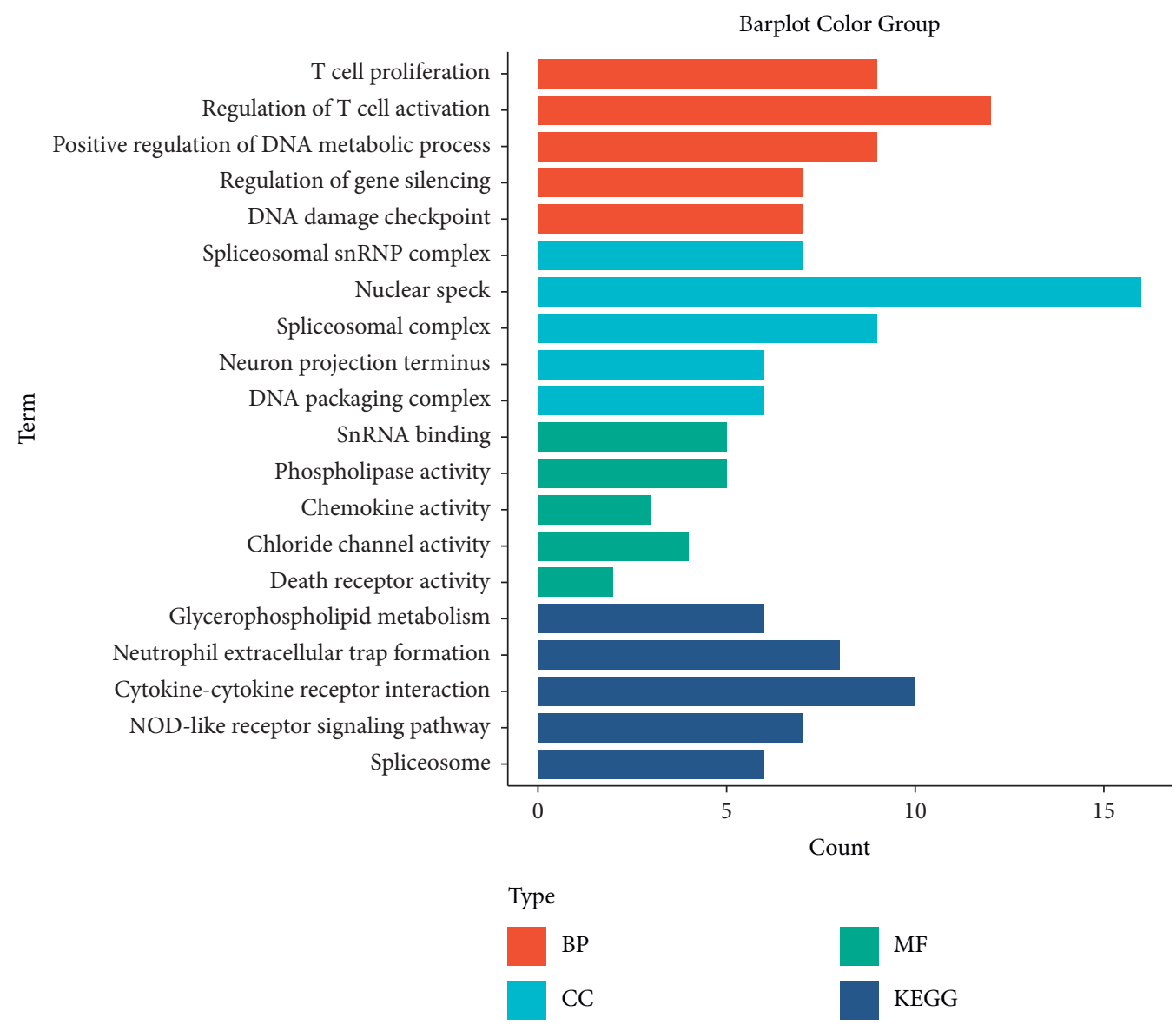

FIGURE 9: Pathway enrichment analysis for the DEGs.

Tanespimycin

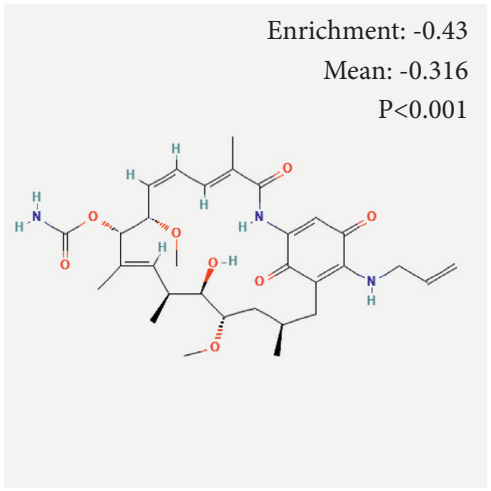

Ursodeoxycholic acid

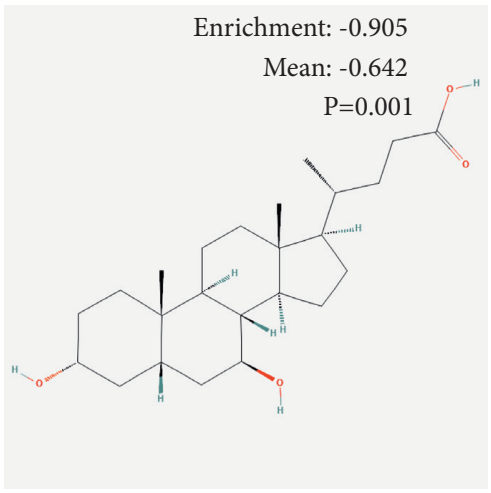

Helveticoside

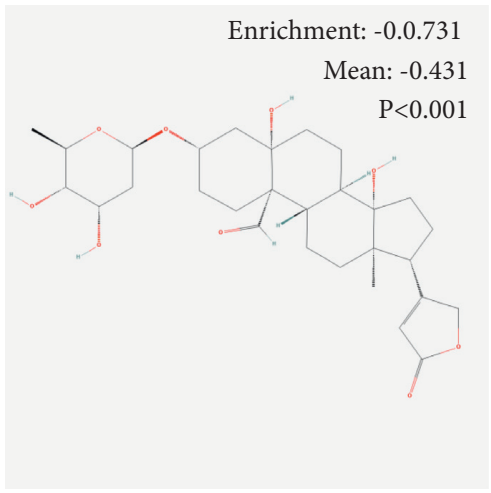

Figure 10: Two-dimensional diagram of the three most significant drugs.

\section{Conclusion}

For the first time in KIRC patients, we identified and validated a predictive model consisting of 7 IR-lncRNAs with independent prognostic significance. In addition, our predictive model may provide a new basis for immunotherapy and immune targets for KIRC. In addition, based on this predictive model, we predicted three small molecule drugs with potential therapeutic value for KIRC treatment and identified genes that intersect with AKI.

\section{Data Availability}

Publicly available datasets were analyzed in this study, and they are available from the Gene Expression Omnibus (GEO) and in The Cancer Genome Atlas (TCGA) database.

\section{Disclosure}

Chenxia Juan and Ye Zhu are co-first authors. 


\section{Conflicts of Interest}

The authors declare that no conflicts of interest exist.

\section{Authors' Contributions}

CJ, YZ, and YC designed the study and wrote the manuscript; YZ, WZ, WH, and XW participated in literature search and data acquisition, analysis, and interpretation. All authors approved the final manuscript. Chenxia Juan and Ye Zhu contributed equally to this work.

\section{Acknowledgments}

This work was supported by grants from the National Natural Science Foundation of China (82100753 and 82174294).

\section{Supplementary Materials}

Table S1: 2683 IRGs from ImmPort Shared Data. Table S2 : IRGs in the red module. Table S3:IRGs in the grey module. Table S4: 63 prognostic IRGs. Table S5 : 206 prognostic IRlncRNAs. Figure S1: volcano plot showing 765 DEGs between high- and low-risk groups. Figure S2: 44 shared DEGs between KIRC and AKI. (Supplementary Materials)

\section{References}

[1] H. Moch, "An overview of renal cell cancer: pathology and genetics," Seminars in Cancer Biology, vol. 23, no. 1, pp. 3-9, 2013.

[2] B. Ljungberg, K. Bensalah, S. Canfield et al., "EAU guidelines on renal cell carcinoma: 2014 update," European Urology, vol. 67, no. 5, pp. 913-924, 2015.

[3] R. E. Gray and G. T. Harris, "Renal cell carcinoma: diagnosis and management," American Family Physician, vol. 99, pp. 179-184, 2019.

[4] E. Jonasch, C. L. Walker, and W. K. Rathmell, "Clear cell renal cell carcinoma ontogeny and mechanisms of lethality," Nature Reviews Nephrology, vol. 17, no. 4, pp. 245-261, 2021.

[5] E. Elinav, R. Nowarski, C. A. Thaiss, B. Hu, C. Jin, and R. A. Flavell, "Inflammation-induced cancer: crosstalk between tumours, immune cells and microorganisms," Nature Reviews Cancer, vol. 13, no. 11, pp. 759-771, 2013.

[6] H. Gonzalez, C. Hagerling, and Z. Werb, "Roles of the immune system in cancer: from tumor initiation to metastatic progression," Genes \& Development, vol. 32, no. 19-20, pp. 1267-1284, 2018.

[7] S. L. Topalian, F. S. Hodi, J. R. Brahmer et al., "Safety, activity, and immune correlates of anti-PD-1 antibody in cancer," New England Journal of Medicine, vol. 366, no. 26, pp. 2443-2454, 2012.

[8] J. K. Shen, G. M. Cote, E. Choy et al., "Programmed cell death ligand 1 expression in osteosarcoma," Cancer Immunology Research, vol. 2, no. 7, pp. 690-698, 2014.

[9] D. C. Hinshaw and L. A. Shevde, "The tumor microenvironment innately modulates cancer progression," Cancer Research, vol. 79, no. 18, pp. 4557-4566, 2019.

[10] I. Vitale, G. Manic, L. M. Coussens, G. Kroemer, and L. Galluzzi, "Macrophages and metabolism in the tumor microenvironment," Cell Metabolism, vol. 30, no. 1, pp. 36-50, 2019.
[11] A. E. Denton, E. W. Roberts, and D. T. Fearon, "Stromal cells in the tumor microenvironment," Stromal Immunology, vol. 1060, pp. 99-114, 2018.

[12] F. Jiang, C. Wu, M. Wang, K. Wei, G. Zhou, and J. Wang, "Multi-omics analysis of tumor mutation burden combined with immune infiltrates in melanoma," Clinica Chimica Acta, vol. 511, pp. 306-318, 2020.

[13] Y. Oya, Y. Hayakawa, and K. Koike, "Tumor microenvironment in gastric cancers," Cancer Science, vol. 111, no. 8, pp. 2696-2707, 2020.

[14] G. Yang, X. Lu, and L. Yuan, "LncRNA: a link between RNA and cancer," Biochimica et Biophysica Acta (BBA) - Gene Regulatory Mechanisms, vol. 1839, no. 11, pp. 1097-1109, 2014.

[15] J. Li, H. Meng, Y. Bai, and K. Wang, "Regulation of lncRNA and its role in cancer metastasis," Oncology Research Featuring Preclinical and Clinical Cancer Therapeutics, vol. 23, no. 5, pp. 205-217, 2016.

[16] W. Wang, Z. Zhao, F. Yang et al., "An immune-related lncRNA signature for patients with anaplastic gliomas," Journal of Neuro-Oncology, vol. 136, no. 2, pp. 263-271, 2018.

[17] G. Wu, Q. Wang, T. Zhu et al., "Identification and validation of immune-related LncRNA prognostic signature for lung adenocarcinoma," Frontiers in Genetics, vol. 12, Article ID 681277, 2021.

[18] X. Li, L. Sun, X. Wang et al., "A five immune-related lncRNA signature as a prognostic target for glioblastoma," Frontiers in Molecular Biosciences, vol. 8, Article ID 632837, 2021.

[19] E. Ma, S. Hou, Y. Wang, X. Xu, Z. Wang, and J. Zhao, "Identification and validation of an immune-related lncRNA signature to facilitate survival prediction in gastric cancer," Frontiers in Oncology, vol. 11, Article ID 666064, 2021.

[20] P. Langfelder and S. Horvath, "WGCNA: an R package for weighted correlation network analysis," BMC Bioinformatics, vol. 9, no. 1, p. 559, 2008.

[21] B. Chen, M. S. Khodadoust, C. L. Liu, A. M. Newman, and A. A. Alizadeh, "Profiling tumor infiltrating immune cells with CIBERSORT," Methods in Molecular Biology, vol. 1711, pp. 243-259, 2018.

[22] M. Suárez-Fariñas, M. A. Lowes, L. C. Zaba, and J. G. Krueger, "Evaluation of the psoriasis transcriptome across different studies by gene set enrichment analysis (GSEA)," PLoS One, vol. 5, no. 4, Article ID e10247, 2010.

[23] N. Chowdhury and C. G. Drake, "Kidney cancer," Urologic Clinics of North America, vol. 47, no. 4, pp. 419-431, 2020.

[24] S. Turajlic, C. Swanton, and C. Boshoff, "Kidney cancer: the next decade," Journal of Experimental Medicine, vol. 215, no. 10, pp. 2477-2479, 2018.

[25] C. Thoma, "Combining targeted and immunotherapy," $\mathrm{Na}$ ture Reviews Urology, vol. 15, no. 5, p. 263, 2018.

[26] J. Lu, Y. Xu, W. Xie et al., "Long noncoding RNA DLGAP1AS2 facilitates Wnt1 transcription through physically interacting with Six3 and drives the malignancy of gastric cancer," Cell Death Discovery, vol. 7, no. 1, p. 255, 2021.

[27] Y. Liang, F. Ye, Y. Wang et al., "DGUOK-AS1 acts as a tumor promoter through regulating miR-204-5p/IL-11 axis in breast cancer," Molecular Therapy - Nucleic Acids, vol. 26, pp. 1079-1091, 2021.

[28] L. Ding, T. Liu, Y. Qu et al., "IncRNA MELTF-AS1 facilitates osteosarcoma metastasis by modulating MMP14 expression," Molecular Therapy - Nucleic Acids, vol. 26, pp. 787-797, 2021.

[29] Y. Cao, W. Tang, and W. Tang, "Immune cell infiltration characteristics and related core genes in lupus nephritis: results from bioinformatic analysis," BMC Immunology, vol. 20, no. 1, p. 37, 2019. 
[30] R. Zhong, D. Chen, S. Cao, J. Li, B. Han, and H. Zhong, "Immune cell infiltration features and related marker genes in lung cancer based on single-cell RNA-seq," Clinical and Translational Oncology, vol. 23, no. 2, pp. 405-417, 2021.

[31] K. Yoshihara, M. Shahmoradgoli, E. Martínez et al., "Inferring tumour purity and stromal and immune cell admixture from expression data," Nature Communications, vol. 4, no. 1, p. 2612, 2013.

[32] M. Kouba and J. Mourot, "A review of nutritional effects on fat composition of animal products with special emphasis on n-3 polyunsaturated fatty acids," Biochimie, vol. 93, no. 1, pp. 13-17, 2011.

[33] P. C. Calder, D. L. Waitzberg, S. Klek, and R. G. Martindale, "Lipids in parenteral nutrition: biological aspects," JPEN Journal of Parenteral and Enteral Nutrition, vol. 44, no. Suppl 1, p. S21, 2020.

[34] M.-A. Dimopoulos, C. S. Mitsiades, K. C. Anderson, and P. G. Richardson, "Tanespimycin as antitumor therapy," Clinical Lymphoma, Myeloma \& Leukemia, vol. 11, no. 1, pp. 17-22, 2011.

[35] H. A. Burris, D. Berman, B. Murthy, and S. Jones, "Tanespimycin pharmacokinetics: a randomized dose-escalation crossover phase 1 study of two formulations," Cancer Chemotherapy and Pharmacology, vol. 67, no. 5, pp. 1045-1054, 2011.

[36] B.-Y. Kim, J. Lee, and N. S. Kim, "Helveticoside is a biologically active component of the seed extract of Descurainia sophia and induces reciprocal gene regulation in A549 human lung cancer cells," BMC Genomics, vol. 16, no. 1, p. 713, 2015.

[37] J.-F. Goossens and C. Bailly, "Ursodeoxycholic acid and cancer: from chemoprevention to chemotherapy," Pharmacology \& Therapeutics, vol. 203, p. 107396, 2019.

[38] S. H. Lee, D. K. Jang, M.-W. Yoo et al., "Efficacy and safety of ursodeoxycholic acid for the prevention of gallstone formation after gastrectomy in patients with gastric cancer," JAMA Surgery, vol. 155, no. 8, p. 703, 2020. 